\title{
Relative Equilibria in the Four-Vortex Problem with Two Pairs of Equal Vorticities
}

\author{
Marshall Hampton, Gareth E. Roberts† Manuele Santoprete ${ }^{\ddagger}$
}

August 22, 2012

\begin{abstract}
We examine in detail the relative equilibria in the four-vortex problem where two pairs of vortices have equal strength, that is, $\Gamma_{1}=\Gamma_{2}=1$ and $\Gamma_{3}=\Gamma_{4}=m$ where $m \in \mathbb{R}-\{0\}$ is a parameter. One main result is that for $m>0$, the convex configurations all contain a line of symmetry, forming a rhombus or an isosceles trapezoid. The rhombus solutions exist for all $m$ but the isosceles trapezoid case exists only when $m$ is positive. In fact, there exist asymmetric convex configurations when $m<0$. In contrast to the Newtonian four-body problem with two equal pairs of masses, where the symmetry of all convex central configurations is unproven, the equations in the vortex case are easier to handle, allowing for a complete classification of all solutions. Precise counts on the number and type of solutions (equivalence classes) for different values of $m$, as well as a description of some of the bifurcations that occur, are provided. Our techniques involve a combination of analysis and modern and computational algebraic geometry.
\end{abstract}

\section{Contents}

1 Introduction $\quad 2$

2 Relative Equilibria 6

2.1 Using mutual distances as coordinates . . . . . . . . . . . . . . 7

2.2 Symmetric configurations . . . . . . . . . . . . . . . . . . 11

2.3 The Albouy-Chenciner equations . . . . . . . . . . . 13

3 Algebraic Techniques

3.1 Gröbner bases and elimination theory . . . . . . . . . . . . . . 14

3.2 A useful lemma for quartic polynomials . . . . . . . . . . . . . 15

3.3 Möbius transformations . . . . . . . . . . . . . . 16

*Dept. of Mathematics and Statistics, University of Minnesota Duluth, mhampton@d.umn.edu

${ }^{\dagger}$ Dept. of Mathematics and Comp. Sci., College of the Holy Cross, groberts@radius.holycross.edu

$\ddagger$ Dept. of Mathematics, Wilfrid Laurier Univerisity, msantoprete@wlu.ca 
4 Special Cases 17

4.1 Symmetric equilibria . . . . . . . . . . . . . . . . . . . . 17

4.2 Vanishing total vorticity $\ldots \ldots \ldots \ldots \ldots \ldots$

5 Collinear Relative Equilibria 18

5.1 Symmetric solutions . . . . . . . . . . . . . . . . . . . . . . 19

5.2 Asymmetric solutions . . . . . . . . . . . . . . . . . . . 20

6 Asymmetric Strictly Planar Relative Equilibria 24

6.1 Eliminating symmetric solutions . . . . . . . . . . . . . . 24 24

6.2 Proof of Lemma 6.1. . . . . . . . . . . . . . . . . . . . 26

6.3 The variety of asymmetric configurations . . . . . . . . . . 29

7 Symmetric Strictly Planar Relative Equilibria 34

7.1 The isosceles trapezoid family . . . . . . . . . . . . . . 34

7.2 Kite configurations: $\lambda^{\prime}<0 \ldots \ldots \ldots \ldots \ldots \ldots \ldots \ldots$

7.3 Kite configurations: $\lambda^{\prime}>0 \ldots \ldots \ldots \ldots \ldots \ldots \ldots$

7.4 The rhombus solutions . . . . . . . . . . . . . . . . 47

\section{Introduction}

The motion of point vortices in the plane is an old problem in fluid mechanics that was first given a Hamiltonian formulation by Kirchhoff [17]. This widely used model provides finite-dimensional approximations to vorticity evolution in fluid dynamics. The goal is to track the motion of the point vortices rather than focus on their internal structure and deformation, a concept analogous to the use of "point masses" in celestial mechanics. As with the Newtonian $n$-body problem, an important class of homographic solutions exist where the configuration is self-similar throughout the motion. Such solutions are described as stationary by O'Neil [21] and are not limited to just relative equilibria (rigid rotations), but also include equilibria, rigidly translating solutions and collapse configurations.

In this paper we focus primarily on the relative equilibria of the four-vortex problem when two pairs of vortices have the same vortex strength. Specifically, if $\Gamma_{i} \in \mathbb{R}-\{0\}$ denotes the vortex strength of the $i$-th vortex, we set $\Gamma_{1}=\Gamma_{2}=1$ and $\Gamma_{3}=\Gamma_{4}=m$, treating $m$ as a real parameter. Our main goal is to classify and describe all solutions as $m$ varies. Four-vortex configurations which are not collinear (nor contain any three vortices which are collinear) can be classified as either concave or convex. A concave configuration has one vortex which is located strictly inside the convex hull of the other three, whereas a convex configuration does not have a vortex contained in the convex hull of the other three vortices.

The symmetry and asymmetry of solutions plays a major role in our analysis. In fact, part of the motivation behind our work was to determine whether symmetry could be proven for this choice of vorticities when $m>0$ and the configuration is assumed to be convex. This question, while solved in the Newtonian four-body problem when the equal 


\begin{tabular}{|c|c|c|}
\hline Shape & $m \in(-1,1]$ & Type of solution (number of) \\
\hline \multirow[t]{5}{*}{ Convex } & $m=1$ & Square (6) \\
\hline & $0<m<1$ & Rhombus (2), Isosceles Trapezoid (4) \\
\hline & $-1<m<0$ & $\begin{array}{c}\text { Rhombus (4) } \\
\text { Asymmetric (8) }\end{array}$ \\
\hline & $-1 / 2<m<0$ & $\mathrm{Kite}_{34}(4)$ \\
\hline & $m^{*}<m<-1 / 2$ & Kite $_{12}(4)$ \\
\hline \multirow[t]{3}{*}{ Concave } & $m=1$ & Equilateral Triangle with Interior Vortex (8) \\
\hline & $0<m<1$ & $\begin{array}{c}\text { Kite }_{34}(8) \\
\text { Asymmetric (8) }\end{array}$ \\
\hline & $-1 / 2<m<0$ & $\mathrm{Kite}_{12}(4)$ \\
\hline \multirow[t]{4}{*}{ Collinear } & $m=1$ & Symmetric (12) \\
\hline & $0<m<1$ & $\begin{array}{l}\text { Symmetric (4) } \\
\text { Asymmetric ( } 8)\end{array}$ \\
\hline & $-1<m<0$ & Symmetric (2) \\
\hline & $-1 / 2<m<0$ & Asymmetric (4) \\
\hline
\end{tabular}

Table 1: The number of relative equilibria equivalence classes for the four-vortex problem with vortex strengths $\Gamma_{1}=\Gamma_{2}=1$ and $\Gamma_{3}=\Gamma_{4}=m$, in terms of $m$ and the type of configuration. The special value $m^{*} \approx-0.5951$ is the only real root of the cubic $9 m^{3}+3 m^{2}+7 m+5$. Kite $_{i j}$ refers to a kite configuration with vortices $i$ and $j$ on the axis of symmetry.

masses are assumed to be opposite each other in a convex central configuration [3, 23], is still open for the case when equal masses are assumed to be adjacent. In contrast, in this paper, we are able to prove that symmetry is required in the case of four vortices with two equal pairs of vorticities. In particular, we show that any convex relative equilibrium with $m>0$, and any concave solution with $m<0$, must have a line of symmetry. For the convex case, the symmetric solutions are a rhombus and an isosceles trapezoid. In the concave case, the symmetric solution is an isosceles triangle with an interior vortex on the axis of symmetry.

A precise count on the number and type of solutions as a function of the parameter $m$ is given in Table 1. A configuration is called a kite if two vortices are on an axis of symmetry and the other two vortices are symmetrically located with respect to this axis. Kite configurations may either be concave or convex. When counting solutions, we use the standard convention from celestial mechanics that solutions which are identical under scaling or rotation are considered equivalent. Note that two solutions identical under a reflection are counted separately. The full set of solutions for $m=2 / 5, m=-1 / 5$ and 
$m=-7 / 10$ (excluding any strictly planar configurations identical under a reflection) are shown in Figure 1.
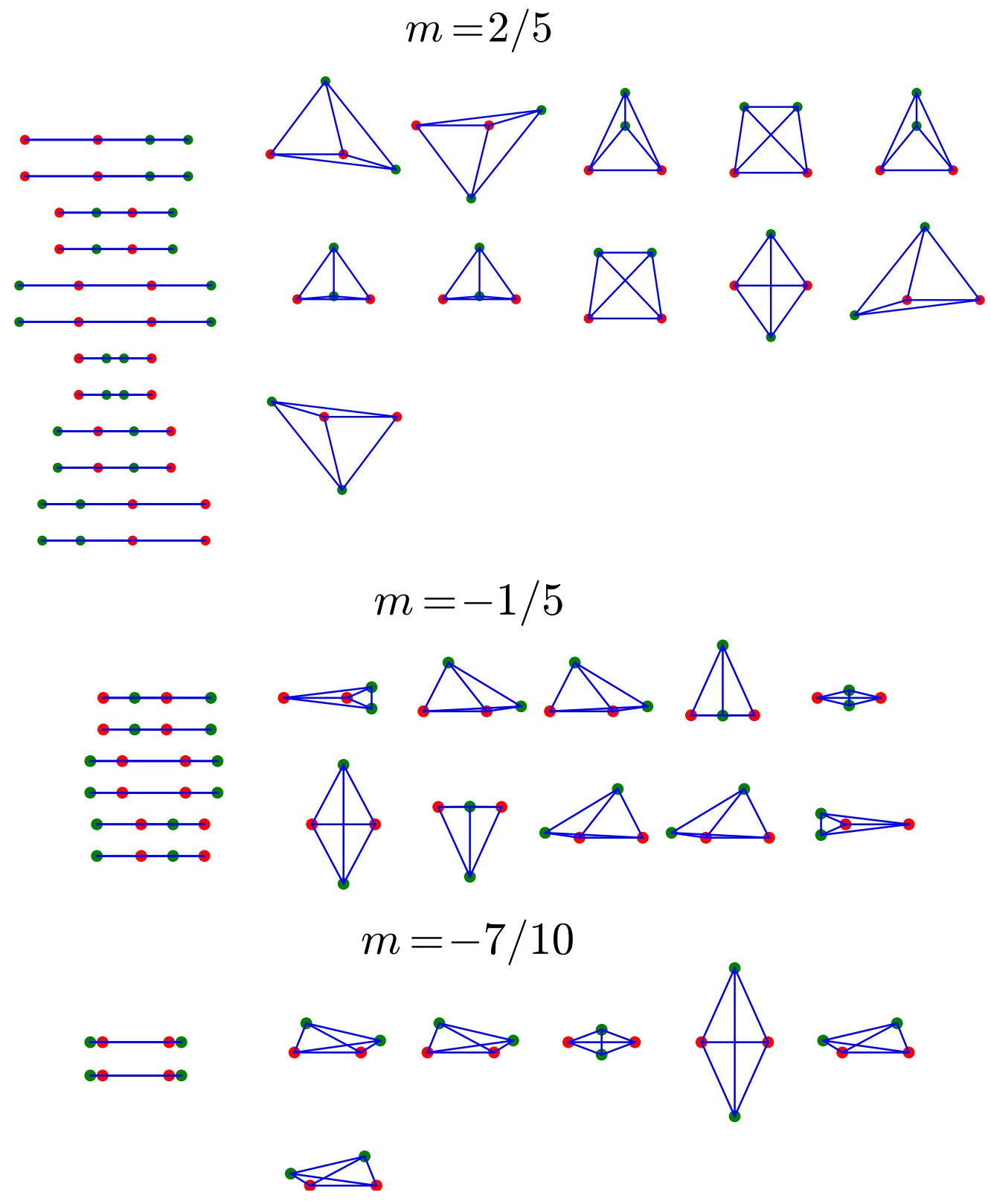

Figure 1: The full set of solutions for $m=2 / 5, m=-1 / 5$ and $m=-7 / 10$. Vortices $\Gamma_{1}=\Gamma_{2}=1$ are denoted by red disks and vortices $\Gamma_{3}=\Gamma_{4}=m$ by green ones.

When $m=1$ (all vortex strengths equal), there are 26 solutions, all symmetric. Allowing for relabeling, there are only three geometrically distinct configurations: a square, an equilateral triangle with a vortex at the center, and a collinear configuration. This 
is different than the Newtonian case, where an additional symmetric, concave solution exists, consisting of an isosceles triangle with an interior body on the axis of symmetry [1]. Part of the reason for the contrast is that the equilateral triangle with central vortex is degenerate when all vortices have the same strength [18, 22]. An interesting bifurcation occurs as $m$ decreases through $m=1$, where the equilateral triangle solution splits into four different solutions. If vortex 3 or 4 is at the center of the triangle, then the solution for $m=1$ bifurcates into two different isosceles triangles with the interior vortex on the line of symmetry. In this case, the larger-strength vortices are on the base of the triangle. If vortex 1 or 2 is at the center of the triangle, the solution branches into two asymmetric concave configurations that are identical under a reflection. In this case, the weaker-strength vortices are on the base of the triangle. Thus, the number of solutions increases from 26 to 34 for the case $0<m<1$. This concurs with Palmore's result in [22], which specifies a lower bound of 34 non-degenerate solutions for the four-vortex problem with positive vorticities.

As $m$ flips sign, there are two interesting bifurcation values at $m=-1 / 2$ and $m=$ $m^{*} \approx-0.5951$, the only real root of the cubic $9 m^{3}+3 m^{2}+7 m+5$. There are 26 solutions for $-1 / 2<m<0$, including a family of convex kite configurations having the negative strength vortices on an axis of symmetry and the positive strength vortices symmetrically located with respect to this axis. Based on the primary result in [3, which is applicable to our problem but only when $m>0$, one might expect this convex kite to have two axes of symmetry, forming a rhombus. However, as demonstrated in Section 7.2, this is not the case. There is also a family of symmetric concave kite configurations having one of the positive strength vortices in the interior of an isosceles triangle formed by the outer three. As $m$ approaches $-1 / 2$, the triangle containing the interior vortex and the base of the outer isosceles triangle limits on an equilateral triangle while the fourth vortex (at the apex of the outer isosceles triangle) heads off to infinity. As $m$ decreases through $-1 / 2$, this family bifurcates into a convex configuration, while the previous convex kite family disappears. This new family of convex kites, with vortices 1 and 2 on the axis of symmetry, exists only for $m^{*}<m<-1 / 2$. In sum, there are 18 solutions for $m^{*}<m<-1 / 2$, and 14 solutions when $m=-1 / 2$ or when $-1<m \leq m^{*}$. The singular bifurcation at $m=-1 / 2$ occurs in part because the leading coefficient of a defining polynomial vanishes. This is likely a consequence of the fact that the sum of three vorticities vanishes when $m=-1 / 2$, a particularly troubling case when attempting to prove finiteness for the number of relative equilibria in the full four-vortex problem (see [16]). The bifurcation at $m=m^{*}$ is a pitchfork bifurcation, as discussed in Section 7.4.

In the next section we define a relative equilibrium and explain how to use mutual distances as variables in the four-vortex problem. In Section 3, we describe the relevant algebraic techniques used to analyze and quantify the number of solutions. Section 4 examines the interplay between symmetry and equality of vorticities in two special cases: equilibria and vanishing total vorticity. Sections 5, 6 and 7 cover the collinear, asymmetric and symmetric cases, respectively, in considerable detail. Throughout our research, symbolic computations (e.g., calculation of Gröbner bases) were performed using Sage [27], Singular [12] and Maple15 ${ }^{\mathrm{TM}}$. 


\section{Relative Equilibria}

We begin with the equations of motion and the definition of a relative equilibrium for the $n$-vortex problem. Let $J=\left[\begin{array}{cc}0 & 1 \\ -1 & 0\end{array}\right]$, and let $\nabla_{j}$ denote the two-dimensional partial gradient with respect to $x_{j}$. A system of $n$ planar point vortices with vortex strengths $\Gamma_{i} \neq 0$ and positions $x_{i} \in \mathbb{R}^{2}$ evolves according to

$$
\Gamma_{i} \dot{x}_{i}=J \nabla_{i} H=-J \sum_{j=1}^{n} \frac{\Gamma_{i} \Gamma_{j}}{r_{i j}^{2}}\left(x_{i}-x_{j}\right), \quad 1 \leq i \leq n
$$

where $H=-\sum_{i<j} \Gamma_{i} \Gamma_{j} \log r_{i j}, r_{i j}=\left\|x_{i}-x_{j}\right\|$, and the prime on the summation indicates omission of the term with $j=i$.

A relative equilibrium motion is a solution of (1) of the form $x_{i}(t)=c+e^{-J \lambda t}\left(x_{i}(0)-c\right)$, that is, a periodic solution given by a uniform rotation with angular velocity $\lambda \neq 0$ around some point $c \in \mathbb{R}^{2}$. Such a solution is possible if and only if the initial positions $x_{i}(0)$ satisfy the equations

$$
-\lambda\left(x_{i}(0)-c\right)=\frac{1}{\Gamma_{i}} \nabla_{i} H=\sum_{j=1}^{n} \frac{\Gamma_{j}}{r_{i j}^{2}}\left(x_{j}(0)-x_{i}(0)\right)
$$

for each $i \in\{1, \ldots, n\}$. Denote $\Gamma=\sum_{i} \Gamma_{i}$ as the total circulation and assume for the moment that $\Gamma \neq 0$. Multiplying the $i$-th equation in $(2)$ by $\Gamma_{i}$ and summing over $i$ shows that the center of rotation $c$ is equivalent to the center of vorticity, $c=\frac{1}{\Gamma} \sum_{i} \Gamma_{i} x_{i}$. If $\Gamma=0$, then we obtain instead that the moment of vorticity $\sum_{i} \Gamma_{i} x_{i}$ must vanish.

Definition 2.1. A set of initial positions $x_{i}(0)$ satisfying equation (2) for each $i \in$ $\{1, \ldots, n\}$ is called a central configuration. The corresponding rigid rotation with angular velocity $\lambda \neq 0$ is called a relative equilibrium. We will often use these two terms interchangeably.

Define the moment of inertia $I$ with respect to the center of vorticity as

$$
I=\frac{1}{2} \sum_{i=1}^{n} \Gamma_{i}\left\|x_{i}-c\right\|^{2}
$$

I measures the size of the system. We can then rewrite equation (2) as

$$
\nabla H+\lambda \nabla\left(I-I_{0}\right)=0
$$

where $\nabla=\left(\nabla_{1}, \ldots, \nabla_{n}\right)$ and $I=I_{0}$. Therefore, $\lambda$ can be viewed as a Lagrange multiplier and any solution of (3) can be interpreted as a critical point of the Hamiltonian $H(x)$ under the condition that $I$ remains constant. Using the homogeneity of the functions $H$ 
and $I$, equation (3) implies that the angular velocity $\lambda$ in a relative equilibrium is given by

$$
\lambda=\frac{L}{2 I} \quad \text { where } \quad L=\sum_{i<j}^{n} \Gamma_{i} \Gamma_{j}
$$

is the total vortex angular momentum. This implies that in the case where all vortex strengths are positive, $\lambda>0$ and the relative equilibrium is rotating in the counterclockwise direction. It is also important to note that for any relative equilibrium with a particular set of vortex strengths $\Gamma_{i}$, we can scale the vorticities by some common factor $\nu$ and maintain the relative equilibrium, but with a new angular velocity $\nu \lambda$. If $\nu<0$, then the sign of $\lambda$ flips, as does the direction of rotation.

Unlike the $n$-body problem, it is possible to have equilibria in the $n$-vortex problem (where $\lambda=0$ ). For $n=4$, explicit solutions can be derived (see [16]). An analysis of the equilibrium solutions in the context of symmetry is presented in Section 4.1 .

\subsection{Using mutual distances as coordinates}

We now consider the case of $n=4$ vortices. Our presentation follows the approach of [24] in describing the work of Dziobek [13] for the Newtonian $n$-body problem. We want to express equation (3) in terms of the mutual distance variables $r_{i j}$. Between four vortices there are six mutual distances, which are not independent if the vortices are planar. In the planar case the distances satisfy the following condition, which can be interpreted as the vanishing of the volume of the tetrahedron formed by the four vortices:

$$
e_{C M}=\left|\begin{array}{ccccc}
0 & 1 & 1 & 1 & 1 \\
1 & 0 & r_{12}^{2} & r_{13}^{2} & r_{14}^{2} \\
1 & r_{12}^{2} & 0 & r_{23}^{2} & r_{24}^{2} \\
1 & r_{13}^{2} & r_{23}^{2} & 0 & r_{34}^{2} \\
1 & r_{14}^{2} & r_{24}^{2} & r_{34}^{2} & 0
\end{array}\right|=0
$$

The matrix in the above determinant is known as the Cayley-Menger matrix.

Hence, planar central configurations are obtained as critical points of

$$
H+\lambda\left(I-I_{0}\right)+\frac{\mu}{32} e_{C M} .
$$

Using the homogeneity of $H, I$ and $e_{C M}$, the value of $\lambda$ in this setup is identical to one given in equation (4). To find $\nabla e_{C M}$ restricted to planar configurations, we use the following important formula

$$
\frac{\partial e_{C M}}{\partial r_{i j}^{2}}=-32 A_{i} A_{j}
$$

where $A_{i}$ is the oriented area of the triangle $T_{i}$ whose vertices are all the vortices except for the $i$-th body. Setting the gradient of (5) equal to zero yields the equations

$$
\frac{\partial H}{\partial r_{i j}^{2}}+\lambda \frac{\partial I}{\partial r_{i j}^{2}}+\frac{\mu}{32} \frac{\partial e_{C M}}{\partial r_{i j}^{2}}=0 .
$$


If $\Gamma \neq 0$, then $I$ can be written in terms of the mutual distances as

$$
I=\frac{1}{2 \Gamma} \sum_{i<j} \Gamma_{i} \Gamma_{j} r_{i j}^{2} .
$$

Using this, we obtain the following equations for a four-vortex central configuration:

$$
\Gamma_{i} \Gamma_{j}\left(r_{i j}^{-2}+\lambda^{\prime}\right)=\sigma A_{i} A_{j}
$$

where $\lambda^{\prime}=-\lambda / \Gamma, \sigma=-2 \mu, I=I_{0}$ and $e_{C M}=0$. If $\Gamma=0$, then a different approach is more useful (see [8, 16] for expositions of the equations). We discuss the role of specific symmetries when $\Gamma=0$ in Section 4.2 .

Assuming $\Gamma \neq 0$, we group the equations in (6) so that when they are multiplied together pairwise, their right-hand sides are identical:

$$
\begin{array}{ll}
\Gamma_{1} \Gamma_{2}\left(r_{12}^{-2}+\lambda^{\prime}\right)=\sigma A_{1} A_{2}, & \Gamma_{3} \Gamma_{4}\left(r_{34}^{-2}+\lambda^{\prime}\right)=\sigma A_{3} A_{4} \\
\Gamma_{1} \Gamma_{3}\left(r_{13}^{-2}+\lambda^{\prime}\right)=\sigma A_{1} A_{3}, & \Gamma_{2} \Gamma_{4}\left(r_{24}^{-2}+\lambda^{\prime}\right)=\sigma A_{2} A_{4} \\
\Gamma_{1} \Gamma_{4}\left(r_{14}^{-2}+\lambda^{\prime}\right)=\sigma A_{1} A_{4}, & \Gamma_{2} \Gamma_{3}\left(r_{23}^{-2}+\lambda^{\prime}\right)=\sigma A_{2} A_{3} .
\end{array}
$$

This yields the well-known Dziobek equations [13, but for vortices:

$$
\left(r_{12}^{-2}+\lambda^{\prime}\right)\left(r_{34}^{-2}+\lambda^{\prime}\right)=\left(r_{13}^{-2}+\lambda^{\prime}\right)\left(r_{24}^{-2}+\lambda^{\prime}\right)=\left(r_{14}^{-2}+\lambda^{\prime}\right)\left(r_{23}^{-2}+\lambda^{\prime}\right) .
$$

From the different ratios of two masses that can be found from the equations in (7) we obtain the following equations:

$$
\begin{aligned}
& \frac{\Gamma_{1} A_{2}}{\Gamma_{2} A_{1}}=\frac{\rho_{23}+\lambda^{\prime}}{\rho_{13}+\lambda^{\prime}}=\frac{\rho_{24}+\lambda^{\prime}}{\rho_{14}+\lambda^{\prime}}=\frac{\rho_{23}-\rho_{24}}{\rho_{13}-\rho_{14}} \\
& \frac{\Gamma_{1} A_{3}}{\Gamma_{3} A_{1}}=\frac{\rho_{23}+\lambda^{\prime}}{\rho_{12}+\lambda^{\prime}}=\frac{\rho_{34}+\lambda^{\prime}}{\rho_{14}+\lambda^{\prime}}=\frac{\rho_{23}-\rho_{34}}{\rho_{12}-\rho_{14}} \\
& \frac{\Gamma_{1} A_{4}}{\Gamma_{4} A_{1}}=\frac{\rho_{24}+\lambda^{\prime}}{\rho_{12}+\lambda^{\prime}}=\frac{\rho_{34}+\lambda^{\prime}}{\rho_{13}+\lambda^{\prime}}=\frac{\rho_{24}-\rho_{34}}{\rho_{12}-\rho_{13}} \\
& \frac{\Gamma_{2} A_{3}}{\Gamma_{3} A_{2}}=\frac{\rho_{13}+\lambda^{\prime}}{\rho_{12}+\lambda^{\prime}}=\frac{\rho_{34}+\lambda^{\prime}}{\rho_{24}+\lambda^{\prime}}=\frac{\rho_{13}-\rho_{34}}{\rho_{12}-\rho_{24}} \\
& \frac{\Gamma_{2} A_{4}}{\Gamma_{4} A_{2}}=\frac{\rho_{14}+\lambda^{\prime}}{\rho_{12}+\lambda^{\prime}}=\frac{\rho_{34}+\lambda^{\prime}}{\rho_{23}+\lambda^{\prime}}=\frac{\rho_{14}-\rho_{34}}{\rho_{12}-\rho_{23}} \\
& \frac{\Gamma_{3} A_{4}}{\Gamma_{4} A_{3}}=\frac{\rho_{14}+\lambda^{\prime}}{\rho_{13}+\lambda^{\prime}}=\frac{\rho_{24}+\lambda^{\prime}}{\rho_{23}+\lambda^{\prime}}=\frac{\rho_{14}-\rho_{24}}{\rho_{13}-\rho_{23}}
\end{aligned}
$$

where $\rho_{i j}=r_{i j}^{-2}$.

Eliminating $\lambda^{\prime}$ from equation (8) and factoring yields the important relation

$$
\left(r_{13}^{2}-r_{12}^{2}\right)\left(r_{23}^{2}-r_{34}^{2}\right)\left(r_{24}^{2}-r_{14}^{2}\right)=\left(r_{12}^{2}-r_{14}^{2}\right)\left(r_{24}^{2}-r_{34}^{2}\right)\left(r_{13}^{2}-r_{23}^{2}\right) .
$$

Assuming the six mutual distances determine an actual configuration in the plane, this equation is necessary and sufficient for the existence of a four-vortex relative equilibrium. The corresponding vortex strengths are then found from the equations in (9). 
Relationships between the lengths of the sides in a four-vortex central configuration follow from the equations in $(9)$ and the signs of the oriented areas $A_{i}$. If the configuration of vortices is concave, precisely three of the oriented areas have the same sign. In the convex case, two oriented areas are positive and two are negative.

The sign of $\lambda^{\prime}=-\lambda / \Gamma$ for a given relative equilibrium is of some interest. Note that scaling the vorticities by any $\nu \in \mathbb{R}-\{0\}$ does not change the value of $\lambda^{\prime}$. If all the vorticities have the same sign, then $\lambda^{\prime}<0$ is assured. However, if the vorticities have different signs, it is possible that $\lambda^{\prime}$ could become positive.

When $\lambda^{\prime}>0$, the equations in $(9)$ imply that $\frac{\Gamma_{i} A_{j}}{\Gamma_{j} A_{i}}>0$ for any choice of indices $i$ and $j$. Taking $\Gamma_{1}=\Gamma_{2}=1$ and $\Gamma_{3}=\Gamma_{4}=m$ with $m<0$, we see that the only possible solutions have $A_{1}, A_{2}>0$ and $A_{3}, A_{4}<0$, or $A_{1}, A_{2}<0$ and $A_{3}, A_{4}>0$. The configuration must be convex with vortices 1 and 2 on one diagonal and vortices 3 and 4 on the other. We show in Section 7.3 that the configuration must have at least one axis of symmetry. There exists a family of convex kite configurations for $m^{*}<m<-1 / 2$ and a family of rhombi (see Section 7.4 for $-1 \leq m<-2+\sqrt{3}$. These are the only possible solutions to our problem having $\lambda^{\prime}>0$.

Proposition 2.2. Suppose we have a concave central configuration with four vortices and $\Gamma \neq 0$.

1. If all the vorticities are positive (negative), then all exterior sides are longer than the interior ones and $1 / \sqrt{-\lambda^{\prime}}$ is less than the lengths of all exterior sides and greater than the lengths of all interior sides.

2. If two of the vorticities are positive and two are negative, then the exterior sides connecting vortices with vorticities of opposite sign and the interior side connecting vortices with vorticities of the same sign have lengths greater than $1 / \sqrt{-\lambda^{\prime}}$, while the remaining sides have length less than $1 / \sqrt{-\lambda^{\prime}}$.

Proof. (1) Let $\Gamma_{4}$ be the interior vorticity and suppose that $A_{4}<0$. Then we have $A_{1}, A_{2}, A_{3}>0$. Assume that $\rho_{34}>-\lambda^{\prime}$. Then, using the equations in (9), we obtain

$$
\rho_{12}, \rho_{13}, \rho_{23}<-\lambda^{\prime}<\rho_{14}, \rho_{24}, \rho_{34},
$$

or

$$
r_{12}, r_{13}, r_{23}>1 / \sqrt{-\lambda^{\prime}}>r_{14}, r_{24}, r_{34}
$$

namely all the exterior edges are longer than the interior ones. On the other hand, if we had assumed $\rho_{34}<-\lambda^{\prime}$, then the inequalities above would be reversed and the configuration could not be realized geometrically since the interior sides cannot all be longer than the exterior sides.

(2) Let $\Gamma_{4}$ be the interior vorticity. Let $\Gamma_{3}, \Gamma_{4}<0$, and $\Gamma_{1}, \Gamma_{2}>0$. Furthermore, suppose that $A_{4}<0$. Then we have $A_{1}, A_{2}, A_{3}>0$. If $\rho_{34}<-\lambda^{\prime}$, then, using the equations in $(9)$, we obtain

$$
\rho_{13}, \rho_{23}, \rho_{34}<-\lambda^{\prime}<\rho_{12}, \rho_{14}, \rho_{24},
$$


or

$$
r_{13}, r_{23}, r_{34}>1 / \sqrt{-\lambda^{\prime}}>r_{12}, r_{14}, r_{24} .
$$

If we had assumed $\rho_{34}>-\lambda^{\prime}$, then the inequalities above would be reversed and the configuration could not be realized geometrically. We show this with a proof by contradiction. Assume $\rho_{34}>-\lambda^{\prime}$. Then $\left|A_{4}\right|<\left|A_{3}\right|$ since the two triangles have one side in common and $r_{24}, r_{14}>r_{23}, r_{13}$. But this is absurd since $T_{4}$ contains $T_{3}$.

Let $\Gamma_{1}$ be the interior vorticity. Let $\Gamma_{3}, \Gamma_{4}<0$, and $\Gamma_{1}, \Gamma_{2}>0$. Furthermore, suppose that $A_{1}<0$. Then we have $A_{2}, A_{3}, A_{4}>0$. If $\rho_{34}>-\lambda^{\prime}$ then, using the equations in (9), we obtain

$$
\rho_{12}, \rho_{23}, \rho_{24}<-\lambda^{\prime}<\rho_{13}, \rho_{14}, \rho_{34},
$$

or

$$
r_{12}, r_{23}, r_{24}>1 / \sqrt{-\lambda^{\prime}}>r_{13}, r_{14}, r_{34} .
$$

If we had assumed $\rho_{34}<-\lambda^{\prime}$ then the inequalities above would be reversed, and the configurations could not be realized geometrically (the proof is similar to the argument in the previous paragraph).

Before we state an analogous theorem for the convex case we recall the following useful geometric lemma:

Lemma 2.3. The combined length of the diagonals of a convex quadrilateral is greater than the combined length of any pair of its opposite sides.

Proof. Order the vortices counterclockwise. Let $o$ be the position vector describing the intersection of the diagonals, and let $r_{i o}=\left\|x_{i}-o\right\|$. Applying the triangle inequality to the triangle of vertices $x_{1}, x_{2}$ and $o$ and to the one of vertices $x_{3}, x_{4}$ and $o$ yields

$$
r_{1 o}+r_{2 o}>r_{12} \text { and } r_{3 o}+r_{4 o}>r_{34} .
$$

Adding these two inequalities together, we obtain $r_{13}+r_{24}>r_{12}+r_{34}$. A similar reasoning can be applied to the remaining two triangles.

Proposition 2.4. Suppose we have a convex central configuration with four vortices, with $\Gamma \neq 0$ and $\lambda^{\prime}<0$.

1. If all the vorticities are positive (negative), then all exterior sides are shorter than the diagonals. Furthermore, the lengths of all exterior sides are less than $1 / \sqrt{-\lambda^{\prime}}$ and the lengths of all the diagonals are greater than $1 / \sqrt{-\lambda^{\prime}}$. The shortest and longest exterior sides have to face each other.

2. If two of the vorticities are positive and adjacent, and two remaining ones are negative, then the exterior sides connecting vortices with vorticities of opposite sign have length less than $1 / \sqrt{-\lambda^{\prime}}$. All the other sides have length greater than $1 / \sqrt{-\lambda^{\prime}}$.

3. If two of the vorticities are positive and opposite, and two are negative, then either all the sides have length less than $1 / \sqrt{-\lambda^{\prime}}$, or all the sides have length greater than $1 / \sqrt{-\lambda^{\prime}}$. 
Proof. (1) The proof is analogous to the one for the Newtonian four-body problem (see [24]).

(2) Order the vortices counterclockwise and let $\Gamma_{1}, \Gamma_{2}>0$ and $\Gamma_{3}, \Gamma_{4}<0$. The sign of the four areas are then $A_{1}>0, A_{3}>0$ and $A_{2}<0, A_{4}<0$. If we assume that $\rho_{34}>-\lambda^{\prime}$, then we find from the equations in $(9)$ that

$$
\rho_{12}, \rho_{13}, \rho_{24}, \rho_{34}<-\lambda^{\prime}<\rho_{14}, \rho_{23},
$$

or

$$
r_{12}, r_{13}, r_{24}, r_{34}>1 / \sqrt{-\lambda^{\prime}}>r_{14}, r_{23} .
$$

On the other hand, if we had assumed that $\rho_{34}<-\lambda^{\prime}$, then the inequalities above would be reversed and, by Lemma 2.3 , the configuration could not be realized geometrically.

(3) Let $\Gamma_{1}, \Gamma_{2}>0$ and $\Gamma_{3}, \Gamma_{4}<0$, and assume the vortices of the same sign are opposite one another. The sign of the four areas are then $A_{1}>0, A_{2}>0$ and $A_{3}<0, A_{4}<0$. If we assume that $\rho_{34}>-\lambda^{\prime}$, then we find from the equations in (9) that

$$
\rho_{12}, \rho_{13}, \rho_{24}, \rho_{34}, \rho_{14}, \rho_{23}>-\lambda^{\prime}
$$

or

$$
r_{12}, r_{13}, r_{24}, r_{34}, r_{14}, r_{23}<1 / \sqrt{-\lambda^{\prime}} .
$$

If $\rho_{34}<-\lambda^{\prime}$, then the inequalities are reversed.

\section{$2.2 \quad$ Symmetric configurations}

One immediate consequence of the Dziobek equations (8) and of equation (10) is that if two mutual distances containing a common vortex are equal (e.g., $r_{12}=r_{13}$ ), then the same equality of distances is true for the excluded vortex (here, $r_{24}=r_{34}$ ). Specifically, if $i, j, k, l$ are distinct indices, then we have

$$
r_{i j}=r_{i k} \text { if and only if } r_{l j}=r_{l k} .
$$

This relation is independent of the vortex strengths although $\Gamma_{j}=\Gamma_{k}$ necessarily must follow. Any configuration satisfying equation (11) has an axis of symmetry containing vortices $i$ and $l$, forming a kite configuration. It may be either convex or concave, but it cannot contain three vortices on a common line due to the equations in (7). Unlike the Newtonian four-body problem, since there is no restriction here on the signs of the vortex strengths, it follows that any kite configuration has a corresponding set of vorticities that make it a relative equilibrium. Assuming the configuration is not an equilateral triangle with a vortex at the center, the vorticities are unique up to a common scaling factor and can be determined by the equations in (9).

Next we consider the case where two mutual distances without a common index are equal. In other words, suppose one of the following three equations holds:

$$
r_{12}=r_{34}, \quad r_{13}=r_{24}, \quad r_{14}=r_{23} .
$$


Then, it does not necessarily follow that another pair of mutual distances must be equal. However, if different pairs of vortices are assumed to be of equal strength, then we can conclude an additional symmetry. This fact will be important in Section 6.1 when verifying that symmetry is required in certain cases.

Lemma 2.5. (Symmetry Lemma) Suppose that we have a strictly planar four-vortex relative equilibrium with $\Gamma_{1}=\Gamma_{2}, \Gamma_{3}=\Gamma_{4}$ and $\Gamma \neq 0$. Then,

$$
r_{13}=r_{24} \quad \text { if and only if } \quad r_{14}=r_{23} .
$$

If either equation in (13) holds, the configuration is convex and has either one or two axes of symmetry. In this case, the configuration is either an isosceles trapezoid with vortices 1 and 2 on one base, and 3 and 4 on the other, or it is a rhombus with vortices 1 and 2 opposite each other.

Proof. Suppose that $r_{13}=r_{24}$. Then, by one of the equations in (7), $\Gamma_{1} \Gamma_{3}\left(r_{13}^{-2}+\lambda^{\prime}\right)=$ $\Gamma_{2} \Gamma_{4}\left(r_{24}^{-2}+\lambda^{\prime}\right)$ implies that

$$
A_{1} A_{3}=A_{2} A_{4}
$$

We also have that

$$
A_{1}+A_{2}+A_{3}+A_{4}=0
$$

since the $A_{i}$ 's are oriented areas. Solving equation f15 for $A_{4}$ and substituting into equation (14) yields the relation

$$
\left(A_{1}+A_{2}\right)\left(A_{2}+A_{3}\right)=0 .
$$

There are two possibilities. First, suppose that $A_{1}=-A_{2}$. Then equation (15) immediately implies $A_{4}=-A_{3}$. The configuration is convex due to the signs of the $A_{i}$ 's and must have the side containing vortices 1 and 2 parallel to the side containing vortices 3 and 4 . Then, by another equation in (7), $\sigma A_{1} A_{4}=\sigma A_{2} A_{3}$ implies that

$$
\Gamma_{1} \Gamma_{4}\left(r_{14}^{-2}+\lambda^{\prime}\right)=\Gamma_{2} \Gamma_{3}\left(r_{23}^{-2}+\lambda^{\prime}\right) .
$$

Since $\Gamma_{1}=\Gamma_{2}$ and $\Gamma_{3}=\Gamma_{4}$, it follows that $r_{14}=r_{23}$. The configuration must be an isosceles trapezoid with congruent legs and diagonals, and base lengths given by $r_{12}$ and $r_{34}$.

Next, suppose that $A_{3}=-A_{2}$. Then, $A_{4}=-A_{1}$ immediately follows from equation (15). The configuration is convex and must have the side containing vortices 1 and 4 parallel to the side containing vortices 2 and 3. Since $r_{13}=r_{24}$, the configuration is either an isosceles trapezoid with $r_{12}=r_{34}$ or is a parallelogram with $r_{14}=r_{23}$. For the isosceles trapezoid, the lengths $r_{13}$ and $r_{24}$ can correspond to either a pair of congruent diagonals or a pair of congruent legs, depending on the ordering of the vortices. However, for the parallelogram, these lengths must correspond to a pair of opposite sides as the diagonals do not have to be congruent.

It turns out that, in this case, the isosceles trapezoid is actually a square. To see this, by an equation in (7), $\sigma A_{1} A_{2}=\sigma A_{3} A_{4}$ implies that

$$
\Gamma_{1}^{2}\left(r_{12}^{-2}+\lambda^{\prime}\right)=\Gamma_{3}^{2}\left(r_{34}^{-2}+\lambda^{\prime}\right) .
$$


Thus, if $r_{12}=r_{34}$, then $\Gamma_{1}=\Gamma_{3}$ and all vortex strengths are equal (the case $\Gamma_{1}=-\Gamma_{3}$ is excluded since $\Gamma \neq 0$ ). In this case, it is straight-forward to show that the isosceles trapezoid reduces to a square (see Section 7.1) and thus $r_{14}=r_{23}$.

In the case of the parallelogram, we have $A_{1}=A_{2}=-A_{3}=-A_{4}$. By the equations in (7), this implies that $r_{13}=r_{14}=r_{23}=r_{24}$ and the configuration is a rhombus with vortices 1 and 2 opposite each other. This proves the forward implication.

The proof in the reverse direction is similar. If $r_{14}=r_{23}$, then we derive $A_{1} A_{4}=A_{2} A_{3}$ from an equation in (7). Taken with equation (15), this yields

$$
\left(A_{1}+A_{2}\right)\left(A_{1}+A_{3}\right)=0 .
$$

As before, the case $A_{1}=-A_{2}$ leads to an isosceles trapezoid with $r_{13}=r_{24}$ and base lengths given by $r_{12}$ and $r_{34}$. The case $A_{1}=-A_{3}$ leads to either the square or a rhombus configuration with vortices 1 and 2 across from each other. In either configuration we deduce that $r_{13}=r_{24}$. This completes the proof.

Remark. 1. Similar results exist if different pairs of vortices are assumed to be equal. For example, if $\Gamma_{1}=\Gamma_{3}$ and $\Gamma_{2}=\Gamma_{4}$, then $r_{12}=r_{34}$ if and only if $r_{14}=r_{23}$.

2. The result is also valid in the Newtonian four-body problem (and for other potentials of the same form) since it only depends on the geometry of the configuration and the inherent structure of the equations in (7).

\subsection{The Albouy-Chenciner equations}

For the remainder of the paper (excluding the special cases discussed in Section 4), we will assume the equality of vortex strengths specified in Lemma 2.5. Specifically, we set $\Gamma_{1}=\Gamma_{2}=1$ and $\Gamma_{3}=\Gamma_{4}=m$, treating $m$ as a real parameter. Without loss of generality, we restrict to the case where $m \in(-1,1]$. The choice $m=-1$ implies that $\Gamma=0$, a special case examined in Section 4.2 .

When $\Gamma \neq 0$, the equations for a relative equilibrium can be written in polynomial form as

$$
f_{i j}=\sum_{k=1}^{n} \Gamma_{k}\left[S_{i k}\left(r_{j k}^{2}-r_{i k}^{2}-r_{i j}^{2}\right)+S_{j k}\left(r_{i k}^{2}-r_{j k}^{2}-r_{i j}^{2}\right)\right]=0,
$$

where $1 \leq i<j \leq n$ and the $S_{i j}$ are given by

$$
S_{i j}=\frac{1}{r_{i j}^{2}}+\lambda^{\prime} \quad(i \neq j), \quad S_{i i}=0 .
$$

These very useful equations are due to Albouy and Chenciner [2] (see also [15] for a nice derivation). They form a polynomial system in the $r_{i j}$ variables after clearing the denominators in the $S_{i j}$ terms. 
Since any relative equilibrium may be rescaled, we will impose the normalization $\lambda^{\prime}=-1$ unless otherwise stated. This usually can be assumed without loss of generality. However, as explained in Section 2.1, for $m<0$ the normalization $\lambda^{\prime}=1$ also needs to be considered. This case is discussed in Sections 7.3 and 7.4. We denote the complete set of polynomial equations determined by $f_{i j}=0$ as $\mathcal{F}$.

From the Albouy-Chenciner equations we can derive a more restrictive set of equations, namely

$$
g_{i j}=\sum_{k=1}^{n} \Gamma_{k} S_{i k}\left(r_{j k}^{2}-r_{i k}^{2}-r_{i j}^{2}\right)=0 .
$$

Since $g_{i j} \neq g_{j i}$, these give 12 distinct equations. We denote the complete set of polynomial equations determined by $g_{i j}=0$ as $\mathcal{G}$, and we will refer to them as the unsymmetrized Albouy-Chenciner equations.

The solutions of the Albouy-Chenciner equation give configurations of all dimensions, but, in the four-vortex problem, they can be specialized to the strictly planar case by adding the three Dziobek equations in (8). Introducing the variables $s_{i j}=r_{i j}^{2}$, these equations can be written as

$$
h_{i j k l}=\left(s_{i j}^{-1}+\lambda^{\prime}\right)\left(s_{k l}^{-1}+\lambda^{\prime}\right)-\left(s_{i k}^{-1}+\lambda^{\prime}\right)\left(s_{j l}^{-1}+\lambda^{\prime}\right)=0,
$$

where $i, j, k$ and $l$ are all distinct indeces. We denote the set of Dziobek equations (with denominators cleared) as $\mathcal{H}$.

\section{Algebraic Techniques}

In this section we briefly describe three of our main algebraic techniques for analyzing solutions to our problem: elimination theory using Gröbner bases, a useful lemma to distinguish when the roots of a quartic are real or complex, and Mobius transformations.

\subsection{Gröbner bases and elimination theory}

We mention briefly some elements from elimination theory and the theory of Gröbner bases that will prove useful in our analysis. For a more detailed exposition see [11.

Let $K$ be a field and consider the polynomial ring $K\left[x_{1}, \ldots, x_{n}\right]$ of polynomials in $n$ variables over $K$. Let $f_{1}, \ldots f_{l}$ be $l$ polynomials in $K\left[x_{1}, \ldots, x_{n}\right]$ and consider the ideal $I=\left\langle f_{1}, \ldots, f_{l}\right\rangle$ generated by these polynomials. Denote $\mathbf{V}(I)$ as the affine variety of $I$.

Definition 3.1. An admissible order $>$ on $K\left[x_{1}, \ldots, x_{n}\right]$ is called a $k$-elimination order if

$$
x_{1}^{a_{1}} \ldots x_{n}^{a_{n}}>x_{k+1}^{b_{k+1}} \ldots x_{n}^{b_{n}}
$$

when $a_{i_{0}}>0$ for some $i_{0} \in\{1, \ldots, k\}$.

A lexicographical (lex) order is an example of $k$-elimination order for all $k$. 
Definition 3.2. The $k$-th elimination ideal $I_{k}$ is the ideal of $K\left[x_{k+1}, \ldots, x_{n}\right]$ defined by

$$
I_{k}=I \cap K\left[x_{k+1}, \ldots, x_{n}\right]
$$

Gröbner bases provide a systematic way of finding elements of $I_{k}$ using the proper term ordering.

Theorem 3.3. (The Elimination Theorem) Let I be an ideal of $K\left[x_{1}, \ldots, x_{n}\right]$ and let $G$ be a Gröbner basis of I with respect to a $k$-elimination order for $k$ where $0 \leq k \leq n$. Then the set

$$
G_{k}=G \cap K\left[x_{k+1}, \ldots, x_{n}\right]
$$

is a Gröbner basis of the $k$-th elimination ideal $I_{k}$.

Gröbner bases also provide a method of determining when an element of $\mathbf{V}\left(I_{k}\right)$ (a partial solution) can be extended to a full solution in $\mathbf{V}(I)$. This can be achieved by repeatedly applying the following theorem.

Theorem 3.4. (The Extension Theorem) Let $K$ be an algebraically closed field and let I be some ideal in $K\left[x_{1}, \ldots, x_{n}\right]$. Let $G_{k-1}$ be a lex Gröbner basis for the elimination ideal $I_{k-1}$ and write each polynomial in $G_{k-1}$ as

$$
g_{i}=h_{i}\left(x_{k+1}, \ldots x_{n}\right) x_{k}^{N_{i}}+\text { terms where } x_{k} \text { has degree }<N_{i},
$$

where $N_{i}>0$ and $h_{i}$ is nonzero. Suppose that $\left(a_{k+1}, \ldots, a_{n}\right)$ is a partial solution in $\mathbf{V}\left(I_{k}\right)$ and that $h_{i}\left(a_{k+1}, \ldots, a_{n}\right) \neq 0$ for some index $i$. Then there exists $a_{k} \in K$ such that $\left(a_{k}, a_{k+1}, \ldots, a_{n}\right) \in \mathbf{V}\left(I_{k-1}\right)$.

\subsection{A useful lemma for quartic polynomials}

The analysis of the collinear case, as well as some strictly planar cases, frequently involves solving a quartic equation whose coefficients are polynomials in $m$. We state here some useful results about quartic equations. Consider the general quartic polynomial $\zeta(x)=$ $a x^{4}+b x^{3}+c x^{2}+d x+e$, with coefficients in $\mathbb{R}$ and $a \neq 0$. We first remove the cubic term of $\zeta$ by the change of variables $x=y-\frac{b}{4 a}$. This produces the polynomial $a \eta(y)$, where $\eta$ is the shifted quartic $\eta(y)=y^{4}+p y^{2}+q y+r$. The discriminant $\Delta$ of any polynomial is a positive constant times the square of the product of all possible differences of roots. The discriminant of $\zeta$ is equivalent to $a^{6}$ times the discriminant of $\eta$. For a general quartic, it is straight-forward to check that if $\Delta>0$, then the roots are either all real or all complex (two pairs of complex conjugates). If $\Delta<0$, then there are two real roots and two complex roots. The roots are repeated if and only if $\Delta=0$.

Let $y_{1}, y_{2}, y_{3}$ and $y_{4}$ be the four roots of $\eta(y)$. By construction, $y_{1}+y_{2}+y_{3}+y_{4}=0$. It follows that

$$
z_{1}=-\left(y_{1}+y_{2}\right)^{2}, \quad z_{2}=-\left(y_{1}+y_{3}\right)^{2}, \quad z_{3}=-\left(y_{1}+y_{4}\right)^{2}
$$


are the roots of the resolvent cubic $\xi(z)=z^{3}-2 p z^{2}+\left(p^{2}-4 r\right) z+q^{2}$. The discriminant of $\xi$ is equivalent to the discriminant of $\eta$. When the discriminant is positive, the resolvent cubic is particularly useful for determining whether the roots of $\eta(y)$ are all real or all complex. Specifically, if the four roots of $\eta(y)$ are real, then $z_{1}, z_{2}$, and $z_{3}$ must be negative and real (or if $q=0$, then $z_{i}=0$ for precisely one $i$ while the remaining $z_{i}$ 's are negative). On the other hand, if the four roots of $\eta(y)$ are complex, then one of the roots of $\xi(z)$ is less than or equal to zero, but two of the roots of $\xi(z)$ must be positive and real. These facts can easily be translated into conditions on the coefficients of the resolvent cubic.

Lemma 3.5. Suppose that the discriminant of a quartic polynomial $\zeta(x)$ is positive and let $\eta(y)=y^{4}+p y^{2}+q y+r$ be the shifted quartic related to $\zeta$. Then, the four distinct roots of $\zeta$ are real if and only if $p<0$ and $p^{2}-4 r>0$.

Proof. Since the discriminant is positive, the roots of $\zeta$ are distinct and either all real or all complex. First suppose that the four roots of $\zeta(x)$ are real. Since $\eta(y)$ is obtained through a simple translation, it follows that the four roots of $\eta(y)$ are distinct and real. Then, the three real roots $z_{1}, z_{2}, z_{3}$ of the resolvent cubic $\xi(z)$ are negative with possibly one root equal to zero (if $q=0$ ). This in turn implies that

$$
\begin{aligned}
z_{1}+z_{2}+z_{3} & =2 p<0 \quad \text { and } \\
z_{1} z_{2}+z_{1} z_{3}+z_{2} z_{3} & =p^{2}-4 r>0 .
\end{aligned}
$$

In the other direction, suppose that $p<0$ and $p^{2}-4 r>0$. This implies that all the coefficients of $\xi(z)$ are positive, and by Descartes' rule of signs, $\xi(z)$ has no positive, real roots. It follows that the four roots of $\eta(y)$ cannot be complex. This shows that the roots of $\zeta(x)$ are real.

\subsection{Möbius transformations}

Möbius transformations are a key tool for isolating roots of polynomials since they allow for a dramatic reduction on the number of variations of signs in the coefficients of a polynomial [5]. Let $\mathcal{P}\left(x_{1}, \ldots, x_{n}\right)$ be a multivariate polynomial in $n$ variables. We use Möbius transformations of the form

$$
x_{i}=\frac{k_{i}^{(2)} y_{i}+k_{i}^{(1)}}{y_{i}+1}
$$

where $k_{i}^{(1)}<k_{i}^{(2)}$ and $i=1, \ldots n$, to obtain changes of variables for $\mathcal{P}\left(x_{1}, \ldots, x_{n}\right)$. The numerator of the rational function thus obtained is a multivariate polynomial in the variables $y_{1}, \ldots, y_{n}$ that restricted to the set $[0, \infty) \times \ldots \times[0, \infty)$ has the same number of roots as $\mathcal{P}\left(x_{1}, \ldots, x_{n}\right)$ restricted to $\left[k_{1}^{(1)}, k_{1}^{(2)}\right) \times \ldots \times\left[k_{n}^{(1)}, k_{n}^{(2)}\right)$.

Möbius transformations can be used to determine partitions of the space into blocks so that, in each block, the coefficients of the polynomials under consideration have a very simple behavior with respect to variations of signs. Therefore, we can use such transformations, together with Descartes' rule of signs, to determine whether a polynomial has a root or does not have a root in a given region of space. 


\section{Special Cases}

In this section, we use some results from [16] to examine two special cases: equilibrium solutions and vanishing total vorticity $(\Gamma=0)$.

\subsection{Symmetric equilibria}

Equilibria are solutions to equation (2) with $\lambda=0$. If we choose coordinates so that the fourth vortex is at the origin, and the third vortex is at $(1,0)$, then the other two vortices in a four-vortex equilibrium must be located at:

$$
\begin{aligned}
& x_{1}=\frac{1}{2\left(\Gamma_{2}+\Gamma_{3}+\Gamma_{4}\right)}\left(2 \Gamma_{4}+\Gamma_{2}, \pm \sqrt{3} \Gamma_{2}\right) \\
& x_{2}=\frac{1}{2\left(\Gamma_{1}+\Gamma_{3}+\Gamma_{4}\right)}\left(2 \Gamma_{4}+\Gamma_{1}, \mp \sqrt{3} \Gamma_{1}\right)
\end{aligned}
$$

where the sign chosen for $x_{2}$ is the opposite of that for $x_{1}$ (see [16] for details).

According to equation (4), for an equilibrium to exist the vorticities must satisfy $L=0$. This is impossible if all the vorticities are equal. If three vorticities are equal, for example with $\Gamma_{1}=\Gamma_{2}=\Gamma_{3}=m$, then $\Gamma_{4}=-m$. We can rescale so that $m=1$ without loss of generality. Using (16) it is easy to see that the only equilibria are equilateral triangles with the opposing vortex located in the center.

Now consider equilibria when $\Gamma_{1}=\Gamma_{2}=1$ and $\Gamma_{3}=\Gamma_{4}=m$. Then, $L=0$ implies $m=-2 \pm \sqrt{3}$. Since we are restricting to $m \in(-1,1]$, we have $m=-2+\sqrt{3} \approx-0.2679$. In this case, the equilibria are rhombi with vortices 1 and 2 opposite each other, and $\frac{r_{34}}{r_{12}}=2-\sqrt{3}=-m$. These rhombi are members of one of the families of rhombi described in Section 7.4 .

Suppose we instead specify the symmetry of the configuration. From $(16)$ it is immediate that there are no collinear equilibria for nonzero vorticities. There are also no isosceles trapezoid equilibria. To see this, consider a trapezoid with $r_{14}=r_{23}$ and $r_{13}=r_{24}$. Then (16) implies that

$$
\frac{\Gamma_{2}}{\Gamma_{2}+\Gamma_{3}+\Gamma_{4}}=\frac{-\Gamma_{1}}{\Gamma_{1}+\Gamma_{3}+\Gamma_{4}},
$$

but there are no real nonzero vorticities satisfying this equation and $L=0$.

Finally, there is the case in which the configuration is a kite (either concave or convex). We choose vortices 1 and 2 to be on an axis of symmetry, so $r_{13}=r_{14}$ and $r_{23}=r_{24}$. Then equation (16) implies that $\Gamma_{3}=\Gamma_{4}$. If we choose any $\Gamma_{2} \neq-2 \Gamma_{4}$ with

$$
\Gamma_{2}=-\frac{2 \Gamma_{1} \Gamma_{4}+\Gamma_{4}^{2}}{\Gamma_{1}+2 \Gamma_{4}}
$$

then $L=0$ is satisfied and there is a kite equilibrium given by (16). If we fix $\Gamma_{4}=1$, then as $\Gamma_{2} \rightarrow-2, \Gamma_{1} \rightarrow \infty$ and the configuration of vortices 2 , 3, and 4 approaches an equilateral triangle while the ordinate of vortex 1 heads off to $\pm \infty$. 


\subsection{Vanishing total vorticity}

When the total vorticity $\Gamma=0$, the analysis of stationary vortex configurations (i.e., those that do not change their shape) requires equations adapted to this special case. For four vorticities we simply apply some of the results from [16].

If three of the vorticities are equal and $\Gamma=0$, we can consider without loss of generality the case $\Gamma_{1}=\Gamma_{2}=\Gamma_{3}=1$ and $\Gamma_{4}=-3$. It is interesting that there are six asymmetric configurations which rigidly translate (three pairs of configurations, within a pair the configurations are reflections of one another), but no symmetric rigidly translating solutions. These configurations can be obtained directly from the equations in [16].

For relative equilibria we use the equations

$$
S_{1}=S_{2}=S_{3}=S_{4}=s_{0}
$$

and

$$
\frac{1}{s_{12}}+\frac{1}{s_{34}}=\frac{1}{s_{13}}+\frac{1}{s_{24}}=\frac{1}{s_{14}}+\frac{1}{s_{23}}
$$

where

$$
S_{i}=\Gamma_{j} s_{i j}+\Gamma_{k} s_{i k}+\Gamma_{l} s_{i l}, \quad\{i, j, k, l\}=\{1,2,3,4\}
$$

and $s_{0}$ is an auxiliary variable. Recall that $s_{i j}=r_{i j}^{2}$. We clear denominators in the equations from (17) to get a polynomial system. Using a Gröbner basis to eliminate $s_{0}$, we find that for $\Gamma_{1}=\Gamma_{2}=\Gamma_{3}=1$ and $\Gamma_{4}=-3$ there are two types of symmetric relative equilibria. The first is the equilateral triangle with vortex 4 at its center. The second type is a concave kite with the three equal vorticities on the exterior isosceles triangle. If we scale the exterior triangle so that its longest side is length 1 , then the base is length $\sqrt{\sqrt{3}-5}$, and the other sides of the interior triangle containing vortex 4 are length $\sqrt{-1+\sqrt{3}}$.

If two pairs of vorticities are equal and $\Gamma=0$, then we have $\Gamma_{1}=\Gamma_{2}=1$ and $\Gamma_{3}=\Gamma_{4}=-1$. In this case there are no rigidly translating solutions. This is somewhat surprising since each pair of opposing vortices would rigidly translate if unperturbed. Using the same equations as we did for the case where three vorticities are equal, we find that there are two relative equilibria, each of which forms a rhombus. These appear in the two families of rhombi described in Section 7.4 , with $\frac{s_{34}}{s_{12}}=3 \pm 2 \sqrt{2}$.

\section{Collinear Relative Equilibria}

Collinear relative equilibria of the four-vortex problem can be studied directly from equation (2) since in this case it reduces to

$$
-\lambda\left(x_{i}-c\right)=\sum_{j \neq i}^{n} \frac{\Gamma_{j}}{x_{j}-x_{i}} \quad \text { for each } i \in\{1,2,3,4\},
$$

where $c, x_{i} \in \mathbb{R}$ rather than $\mathbb{R}^{2}$. Clearing denominators from these equations yields a polynomial system. Rather than fix $\lambda$ or $c$, we use the homogeneity and translation 
invariance of the system and set $x_{3}=-1$ and $x_{4}=1$. As specified earlier, we set $\Gamma_{1}=\Gamma_{2}=1$ and $\Gamma_{3}=\Gamma_{4}=m \in(-1,1]$, treating $m$ as a parameter.

\subsection{Symmetric solutions}

Given our setup, symmetric configurations correspond to solutions where either $x_{1}=-x_{2}$ or $r_{12}=2$. Both cases are simple enough to analyze using Gröbner bases.

Case 1: $x_{1}=-x_{2}$

In this case the center of vorticity $c$ is at the origin. The solutions are the roots of an even quartic polynomial in $x_{1}$, given by $m x_{1}^{4}-5(m+1) x_{1}^{2}+1$. The discriminant of this quartic changes sign at $m=0$ and there are four real roots for $m>0$ but only two when $m<0$. The result is that for any $m \in(-1,1]$, there is a collinear relative equilibrium with

$$
x_{1}=-x_{2}= \pm \sqrt{\frac{5 m+5-\sqrt{25 m^{2}+46 m+25}}{2 m}}
$$

(with $x_{1}=-x_{2}= \pm 1 / \sqrt{5}$ when $m=0$ ). This solution has vortices $x_{1}$ and $x_{2}$ symmetrically located between vortices $x_{3}$ and $x_{4}$, and as $m \rightarrow-1^{+}$, the inner vortices approach the outer vortices (with collision at $m=-1$.) For $m \in(0,1],\left|x_{1}\right|$ decreases monotonically in $m$ from $1 / \sqrt{5} \approx 0.4472$ to $\sqrt{3}-\sqrt{2} \approx 0.3178$, while for $m \in(-1,0),\left|x_{1}\right|$ decreases monotonically in $m$ from 1 to $1 / \sqrt{5}$.

There is an additional collinear relative equilibrium if $m \in(0,1]$ given by

$$
x_{1}=-x_{2}= \pm \sqrt{\frac{5 m+5+\sqrt{25 m^{2}+46 m+25}}{2 m}} .
$$

In this case, the vortices $x_{1}$ and $x_{2}$ are symmetrically located outside vortices $x_{3}$ and $x_{4}$, and their positions approach $\pm \infty$ as $m \rightarrow 0^{+}$. The value of $\left|x_{1}\right|$ decreases monotonically in $m$ from $\infty$ to $\sqrt{3}+\sqrt{2} \approx 3.1462$.

Case 2: $r_{12}=2$

In this case the center of vorticity $c$ is not necessarily located at the origin; however, the configuration will be symmetric about some fixed point equidistant from both the inner and outer pairs of vortices. Since $\left|x_{1}-x_{2}\right|=2$ and $x_{1}=-x_{2}$ together imply $\left|x_{2}\right|=1$ (collision), the two cases are distinct. If $m \neq 1$, a solution for case 2 will have the inner and outer pair of vortices having different circulations. It turns out that this case is impossible when $m \neq 1$.

To see this, we consider the polynomials obtained from system (18) along with $x_{1}-x_{2}-2$ and $u\left(x_{1}+x_{2}\right)-1$ (to eliminate solutions from Case 1). Computing a lex Gröbner basis for this set of polynomials quickly yields $m=1$. The same result is obtained when using 
$x_{1}-x_{2}+2$. Moreover, in each computation, a fourth-degree polynomial in $x_{2}$ is obtained that provides the exact solution for the special case $m=1$.

In sum, there are no solutions with $r_{12}=2$ unless $m=1$. When $m=1$, there are 8 solutions with $r_{12}=2$ given by $\left(x_{1}, x_{2}\right)=$

$$
(1 \pm \sqrt{2} \pm \sqrt{6},-1 \pm \sqrt{2} \pm \sqrt{6}) \quad \text { and } \quad(-1 \pm \sqrt{2} \pm \sqrt{6}, 1 \pm \sqrt{2} \pm \sqrt{6})
$$

where the signs in a particular ordered pair are chosen to have the same pattern in each coordinate (e.g., $(1+\sqrt{2}-\sqrt{6},-1+\sqrt{2}-\sqrt{6}))$. It is straight-forward to check that each of these 8 solutions is a scaled version of a solution found in Case 1. Specifically, for each of the solutions listed in $(20)$ as well as the solutions obtained in Case 1 for $m=1$, there is a rescaling, translation and relabeling of the vortices that maps the solution onto

$$
x_{3}=-1, x_{2}=-\sqrt{3}+\sqrt{2}, x_{1}=\sqrt{3}-\sqrt{2}, x_{4}=1,
$$

a solution obtained from equation (19) when $m=1$. We note that solution (21) can be rescaled to coincide with the roots of the Hermite polynomial $H_{4}$, as expected (see [4]).

\section{$5.2 \quad$ Asymmetric solutions}

To locate any asymmetric solutions, we introduce the variables $u$ and $v$ along with the equations

$$
u\left(x_{1}+x_{2}\right)-1 \quad \text { and } \quad v\left(x_{1}-x_{2}\right)-1 .
$$

Adding these two equations to the original polynomial system obtained from (18), we compute a Gröbner basis $G_{\text {col }}$ with respect to the lex order where $c>\lambda>u>v>x_{1}>$ $x_{2}>m$. This basis has 15 elements, the first of which is an even, 8th-degree polynomial in $x_{2}$ with coefficients in $m$, and a basis for the elimination ideal $I_{5}=I \cap \mathbb{C}\left[x_{2}, m\right]$. Introducing the variable $w=x_{2}^{2}$, this polynomial is given by

$$
\begin{aligned}
\zeta(w)= & m^{2}(m+2)(1+2 m)^{2} w^{4}-4 m\left(15 m^{4}+61 m^{3}+91 m^{2}+61 m+15\right) w^{3} \\
& +\left(300 m^{5}+1508 m^{4}+2910 m^{3}+2696 m^{2}+1188 m+200\right) w^{2} \\
& -4(5 m+4)\left(25 m^{4}+127 m^{3}+231 m^{2}+175 m+45\right) w+(m+2)^{3} .
\end{aligned}
$$

If we eliminate $x_{2}$ instead of $x_{1}$, the same polynomial is obtained with $w=x_{1}^{2}$. The discriminant of $\zeta$ is

$$
1048576(m+2)^{2}(m+1)^{6}(1+2 m)\left(25 m^{2}+58 m+25\right)^{3} m^{2}\left(q_{u}(m)\right)^{2}
$$

where $q_{u}(m)=2 m^{5}-16 m^{4}-96 m^{3}-162 m^{2}-108 m-25$. For $m \in(0,1]$, the discriminant is strictly positive. However, on the interval $[-1,0]$, the discriminant vanishes at six different $m$-values. In increasing order, these values are

$$
-1, m_{0} \approx-0.6833, m_{1} \approx-0.6066, m_{2} \approx-0.5721,-1 / 2,0
$$

The values $m_{0}$ and $m_{1}$ are roots of the quintic polynomial $q_{u}$, and $m_{2}=(-29+6 \sqrt{6}) / 25$ is the largest root of the quadratic $25 m^{2}+58 m+25$. For $m=-1, \zeta$ has a repeated root 
at 1 of multiplicity four. At $m=-1 / 2, \zeta$ becomes a cubic polynomial with roots -3 and 1 (multiplicity 2). Thus, the values $m=-1$ and $m=-1 / 2$ have no physical solutions (just collisions). For $m=0$, the quartic reduces to a quadratic, and we have two possible solutions at $w=(9 \pm 4 \sqrt{5}) / 5$ which correspond to limiting configurations as $m \rightarrow 0^{+}$. The other three values where the discriminant vanishes also have no physical solutions, as we show below.

Lemma 5.1. The quartic polynomial $\zeta(w)$ has precisely four positive real roots for $m \in$ $(0,1]$ and two positive real roots for $m \in(-1 / 2,0)$. For $m \in(-1,-1 / 2)$, there are no positive roots except when $m=m_{0}$, where there is one positive, repeated root of multiplicity two.

Proof. Using Lemma 3.5, let $\eta$ be the shifted quartic of $\zeta(w)$. From $\eta$, the key quantities $p$ and $p^{2}-4 r$ are found to be

$$
p=-\frac{2\left(25 m^{2}+58 m+25\right)\left(3 m^{4}+14 m^{3}+45 m^{2}+54 m+19\right)(m+1)^{2}}{m^{2}(m+2)^{2}(1+2 m)^{4}}
$$

and

$$
p^{2}-4 r=\frac{16\left(25 m^{2}+58 m+25\right)(m+1)^{2} t(m)}{m^{4}(m+2)^{4}(1+2 m)^{8}}
$$

where

$$
\begin{aligned}
t(m)= & 75 m^{12}+896 m^{11}+5528 m^{10}+23492 m^{9}+77272 m^{8}+197816 m^{7}+376194 m^{6} \\
& +509968 m^{5}+478976 m^{4}+302388 m^{3}+121964 m^{2}+28320 m+2875 .
\end{aligned}
$$

For $m \in(0,1]$, since the discriminant is positive, $p<0$ and $p^{2}-4 r>0$, Lemma 3.5 applies and the roots of $\zeta(w)$ are all real. The fact that they are all positive follows from Descartes' rule of signs since $\zeta(w)$ has four sign changes while $\zeta(-w)$ has none. For $m \in(-1 / 2,0)$, the discriminant remains positive and using Sturm's theorem, one can show that $p<0$ and $p^{2}-4 r>0$ both continue to hold. It follows that $\zeta(w)$ has four real roots in this case as well. However, two of the roots are positive and two are negative since both $\zeta(w)$ and $\zeta(-w)$ each have two sign changes.

For the case $m \in\left(m_{2},-1 / 2\right)$, the discriminant is negative and thus there are precisely two real roots. To show that the real roots are both negative, we note that at $m=m_{2}$, the quartic $\zeta$ has a repeated root of multiplicity four at $w_{2}=-(11+4 \sqrt{6}) / 5<0$. We then can check that $\zeta\left(w_{2}\right)$ as a function of $m$ is strictly negative for $m \in\left(m_{2},-1 / 2\right)$. Since the leading coefficient of $\zeta$ is positive and since $\zeta(0)=(m+2)^{3}>0$, it follows that both real roots must be negative in this case. There are no positive roots at $m=m_{2}$ since $w_{2}<0$ is the only root.

For the interval $m \in\left(-1, m_{2}\right)$, the discriminant is positive except when it vanishes at $m_{0}$ and $m_{1}$. However, either $p>0$ or $p^{2}-4 r<0$ (or both) for $m$-values on this interval. Consequently, by Lemma 3.5, if $m \in\left(-1, m_{2}\right)-\left\{m_{0}, m_{1}\right\}$, then $\zeta$ has four complex roots.

It remains to check the two cases $m=m_{0}$ and $m=m_{1}$, where the discriminant of $\zeta$ vanishes. Since the roots are complex in a neighborhood of each parameter value, one 
has to check how the roots become repeated as the key parameter value is approached. It turns out that in each case, a pair of complex conjugate roots meet on the real axis. To see this, we use Descartes' rule of signs to check that the resolvent cubic $\xi$ has one negative root and a repeated positive root at both $m=m_{0}$ and $m=m_{1}$. It follows that one pair of complex conjugate roots has merged into a real root since any other scenario would require that zero be a double root of the resolvent cubic. Because the quintic $q_{u}(m)$ is squared in the discriminant, the roots return to being two pairs of complex conjugates after $m$ passes through these special parameter values.

To determine whether the repeated real root of $\zeta$ is positive or negative, we note that at either value $m=m_{0}$ or $m=m_{1}$, the shifted quartic $\eta(w)$ has the form

$$
\left(w-r_{1}\right)^{2}\left[\left(w+r_{1}\right)^{2}+r_{2}^{2}\right]=w^{4}+\left(r_{2}^{2}-2 r_{1}^{2}\right) w^{2}-2 r_{1} r_{2}^{2} w+r_{1}^{2}\left(r_{1}^{2}+r_{2}^{2}\right) .
$$

Thus, the coefficient of the linear term of $\eta$ can be used to determine the sign of the repeated real root. Using Sturm's theorem, one can check that this coefficient is negative at $m=m_{0}$ resulting in a positive value of $r_{1}$. Then, since $\eta(w)=\zeta(w+\hat{c})$, where $\hat{c}>0$ at $m=m_{0}$, we know that the repeated real root of $\zeta$ when $m=m_{0}$ is positive. To find this root exactly, we calculate the resultant of $\zeta(w)$ and $\frac{d}{d w}(\zeta)$ with respect to $m$. This produces a quintic in $w$ given by

$$
5 w^{5}-53 w^{4}+98 w^{3}+198 w^{2}+9 w-1 .
$$

The largest root of this quintic, which is approximately 6.9632775 , is the positive, repeated root of $\zeta(w)$ when $m=m_{0}$. For the special case $m=m_{1}$, the derivative of $\zeta(w)$ is a cubic with only one real negative root. Consequently, the repeated real root in this case is negative.

While it is interesting to discover that the special parameter value $m=m_{0}$ has a unique solution, it cannot lead to a physical solution to the problem because $x_{1}$ necessarily has to be a different root of $\zeta$ (since we are excluding the symmetric solutions). But the other roots of $\zeta$ are complex. The fact that $x_{1}$ is complex is also confirmed by solving one of the polynomials in the Gröbner basis $G_{\text {col }}$ for $x_{1}$ when $m=m_{0}$ and $x_{2}=\sqrt{6.9632775}$.

We summarize our findings for the collinear case in the following theorem.

Theorem 5.2. For the case $m \in(0,1]$, there are 12 collinear solutions, one for each possible ordering of the vortices. If $m=1$, all solutions are symmetric and geometrically equivalent to the same configuration; otherwise, there are 4 symmetric solutions and 8 asymmetric solutions. For $m \in(-1 / 2,0)$, there are a total of 6 solutions, 2 symmetric and 4 asymmetric, while for $m \in(-1,-1 / 2]$, there are only 2 symmetric solutions and no asymmetric solutions.

Proof. The second polynomial in the Gröbner basis $G_{\text {col }}$, denoted $g_{2}$, is in the elimination ideal $I_{4}=I \cap \mathbb{C}\left[x_{1}, x_{2}, m\right]$ and is linear in the variable $x_{1}$. The coefficient of $x_{1}$ is found to be $8(2 m+1)(m+2)^{2} \cdot q_{u}(m)$, which is nonzero for $m \in(-1 / 2,1]$. By the Extension Theorem, we can extend any solution $\left(x_{2}, m\right)$ of $\zeta=0$ to a partial solution $\left(x_{1}, x_{2}, m\right)$ in 
$\mathbf{V}\left(I_{4}\right)$. Moreover, since $g_{2}$ is linear in $x_{1}$, there is at most one such solution, and $x_{1}$ must be real.

Next, we check that the value of $x_{1}$ is distinct from the value of $x_{2}$ (excluding collision) and $-x_{2}$ (excluding the symmetric solutions.) This is accomplished by substituting $x_{1}=x_{2}$ into $g_{2}$ and then computing the resultant of this polynomial with $\zeta\left(x_{2}, m\right)$. A polynomial in $m$ is thus obtained and it is easily checked that this polynomial has no roots for $m \in$ $(-1 / 2,1]$. Therefore, the value of $x_{1}$ obtained by solving $g_{2}=0$ is distinct from $x_{2}$. A similar calculation, using the substitution $x_{1}=-x_{2}$ in $g_{2}$, shows that $x_{1} \neq-x_{2}$ as well.

At this point, the Extension Theorem can be applied four more times using four of the basis polynomials in $G_{\text {col }}$ that are linear in the variables $v, u, \lambda$ and $c$, respectively, each having nonzero leading coefficients. Thus, fixing an $m \in(-1 / 2,1]$, for each positive root of $\zeta(w)$, we obtain two possible values of $x_{2}$, each of which extends uniquely to a full, real, asymmetric solution of our problem. The precise count on the number of solutions for each case then follows directly from Lemma 5.1 and Section 5.1 .

The fact that there is one collinear relative equilibrium for each ordering of the vortices when $m>0$ follows from a straight-forward generalization of a well-known result in the Newtonian $n$-body problem due to Moulton [20]. In brief, for each of the $n$ ! connected components of the phase space for the collinear $n$-vortex problem, there is a unique minimum of $H$ restricted to the ellipsoid $I=I_{0}$ (see p. 33 of [19] or Section 6.1 of [21] for details). Each such minimum is a collinear relative equilibrium. Identifying solutions equivalent under a $180^{\circ}$ rotation of the plane gives a final count of $n ! / 2$.

Theorem 5.3. When $m \in(-1,0)$, the signs of the vorticities in a collinear relative equilibrium must be arranged as +--+ (symmetric case only), or as +-+- or -+-+ (when asymmetric solutions exist). Therefore, when $m<0$, it is not possible to have a collinear solution where both pairs of vortices with the same strength are adjacent to each other.

Proof. The symmetric case has already been analyzed at the start of this section. For the asymmetric case, we note that

$$
\zeta(1)=-128(1+2 m)(m+2)^{2}(m+1)^{2}
$$

is strictly negative when $m \in(-1 / 2,0)$. We also note that $\zeta(0)>0$ and the leading coefficient of $\zeta(w)$ is always positive. Since $\zeta$ has only two positive roots for $m \in(-1 / 2,0)$, it follows that one root is less than one and the other is larger than one. (The two roots approach one as $m \rightarrow-1 / 2^{+}$.) The values of $x_{1}^{2}$ and $x_{2}^{2}$ must each be roots of $\zeta(w)$. Because we are only considering asymmetric solutions, we have that $\left|x_{1}\right|<1$ and $\left|x_{2}\right|>1$ or vice versa. Therefore, when $m \in(-1 / 2,0)$, the only possible asymmetric orderings of vortices have signs arranged as +-+- or -+-+ . 


\section{Asymmetric Strictly Planar Relative Equilibria}

\subsection{Eliminating symmetric solutions}

In this section we study strictly planar relative equilibria that do not have a line of symmetry. A major result proved here is that any convex solution with $m>0$ or any concave solution with $m<0$ must contain a line of symmetry. We prove this by saturating the Gröbner basis in order to eliminate any symmetric solutions, and then showing the resulting system has no real solutions.

Let $\widetilde{\mathcal{F}}$ and $\widetilde{\mathcal{G}}$ be the Albouy-Chenciner and the unsymmetrized Albouy-Chenciner equations in terms of $s_{i j}=r_{i j}^{2}$, respectively, with $\lambda^{\prime}=-1, \Gamma_{1}=\Gamma_{2}=1$, and $\Gamma_{3}=\Gamma_{4}=m \neq-1$. Let $\tilde{e}_{C M}$ be the Cayley-Menger determinant written in terms of $s_{i j}=r_{i j}^{2}$ and let $\widetilde{\mathcal{H}}$ be the Dziobek equations with $\lambda^{\prime}=-1$. The polynomials $\widetilde{\mathcal{F}}, \widetilde{\mathcal{G}}, \tilde{e}_{C M}$, and $\widetilde{\mathcal{H}}$ belong to the polynomial ring $\mathbb{C}\left[m, s_{12}, s_{13}, s_{14}, s_{23}, s_{24}, s_{34}\right]$.

We begin by finding a Gröbner basis for the ideal $I_{s}=\left\langle\widetilde{\mathcal{F}}, \widetilde{\mathcal{G}}, \tilde{e}_{C M}, \widetilde{\mathcal{H}}\right\rangle$. In order to accomplish this we first find a Gröbner basis $G_{J_{s}}$ for the ideal $J_{s}=\left\langle\widetilde{\mathcal{F}}, \widetilde{\mathcal{G}}, \tilde{e}_{C M}\right\rangle$ and then we compute a Gröbner basis $G_{I_{s}}$ for $I_{s}=\left\langle G_{J_{s}}, \widetilde{\mathcal{H}}\right\rangle$. At this stage we saturate with respect to the variables $s_{13}, s_{14}$, and $s_{24}$, to eliminate possible solutions where one of the mutual distances has zero length. We also saturate with respect to $\left(s_{13}-s_{24}\right),\left(s_{14}-s_{23}\right),\left(s_{13}-s_{14}\right)$, $\left(s_{23}-s_{24}\right),\left(s_{13}-s_{23}\right)$, and $\left(s_{14}-s_{24}\right)$. Due to Lemma 2.5 and equation (11), saturating with respect to these differences is equivalent to eliminating any symmetric solutions. We denote the resulting Gröbner basis as $\widetilde{G}_{I_{s}}$.

Computing $\widetilde{G}_{I_{s}}$ with respect to an elimination order that eliminates all the variables except $s_{12}$ and $s_{34}$ yields the following system of two equations in two unknowns:

$$
s_{34} m+s_{12}-m-1=0, \quad s_{12}^{2}-2 s_{12} s_{34}+s_{34}^{2}-1=0 .
$$

This system has the two solutions

$$
\left(s_{12}, s_{34}\right)=\left(\frac{2 m+1}{m+1}, \frac{m}{m+1}\right), \quad\left(s_{12}, s_{34}\right)=\left(\frac{1}{m+1}, \frac{m+2}{m+1}\right),
$$

where one solution is sent to the other one by the transformation $m \rightarrow \frac{1}{m}$ and $s_{12} \leftrightarrow s_{34}$. If we compute the Gröbner basis of $\tilde{G}_{I_{s}}$ with respect to an elimination order that eliminates all but one $s_{i j}=x$, we obtain a polynomial that is the product of the two polynomials

$$
\begin{aligned}
p_{1}= & 4\left(m^{2}+2 m+1\right) x^{4}-4\left(5 m^{2}+8 m+3\right) x^{3}+2\left(16 m^{2}+21 m+7\right) x^{2} \\
& -2\left(10 m^{2}+11 m+3\right) x+4 m^{2}+4 m+1, \quad \text { and } \\
p_{2}= & 4\left(m^{2}+2 m+1\right) x^{4}-4\left(3 m^{2}+8 m+5\right) x^{3}+2\left(7 m^{2}+21 m+16\right) x^{2} \\
& -2\left(3 m^{2}+11 m+10\right) x+m^{2}+4 m+4,
\end{aligned}
$$

where $x$ is one of $s_{13}, s_{14}, s_{23}$ or $s_{24}$. For the case when $s_{12}$ or $s_{34}$ is the only variable not eliminated, we obtain the products

$$
\left(m s_{12}+s_{12}-1\right)\left(m s_{12}+s_{12}-2 m-1\right), \quad \text { or }
$$




$$
\left(m s_{34}+s_{34}-m\right)\left(m s_{34}+s_{34}-m-2\right),
$$

respectively. All the Gröbner basis computations were performed using Singular [12] and Sage [27].

Analyzing the polynomials $p_{1}$ and $p_{2}$ (which we do in the next section), one can prove the following:

Lemma 6.1. For any $m \in(-1,1)$, the polynomial $p_{1}$ has no real positive roots. For any $m \in(-1,1)$, the polynomial $p_{2}$ has four positive distinct real roots except for $m=0$, where one of the roots is repeated (at 1 ). If $m \in[0,1]$, each root of $p_{2}$ lies in one of the intervals $J_{1}=\left[0, \frac{1}{2}\right], J_{2}=\left[\frac{1}{2}, 1\right], J_{3}=\left[1, \frac{3}{2}\right]$, and $J_{4}=\left[\frac{3}{2}, 5\right]$. If $m \in(-1,0)$, each root of $p_{2}$ lies in one of the intervals $K_{1}=\left[0, \frac{1}{2}\right], K_{2}=\left[\frac{1}{2}, 1\right], K_{3}=\left[1, \frac{m+2}{m+1}\right]$ and $K_{4}=\left[\frac{m+2}{m+1}, \infty\right]$.

Using Lemma 6.1, we obtain the following fundamental result:

Theorem 6.2. Let $\mathbf{x}$ be a strictly planar relative equilibrium of the four-vortex problem with vorticities $\Gamma_{1}=\Gamma_{2}=1$ and $\Gamma_{3}=\Gamma_{4}=m$.

1. For any $m>0$, every convex relative equilibrium $\mathbf{x}$ has a line of symmetry, and the only possible strictly planar asymmetric configurations are concave with the two vortices of smaller strength lying on the exterior triangle. If $m=1$, all solutions contain a line of symmetry.

2. For any $m<0$, every concave relative equilibrium $\mathbf{x}$ has a line of symmetry, and the only possible strictly planar asymmetric configurations are convex with equal-strength vortices necessarily adjacent.

Proof. First note that the case $\lambda^{\prime}>0$ is necessarily excluded in the hypotheses of the theorem. If $m>0, \lambda^{\prime}=-\lambda / \Gamma<0$ is guaranteed. If $m<0$ and $\lambda^{\prime}>0$, then the configuration must be convex. Hence, we can assume that $\lambda^{\prime}=-1$.

If we compute a Gröbner basis of $\tilde{G}_{I_{s}}$ that eliminates all the $s_{i j}$ variables except $s_{12}$ and $s_{13}$, we obtain several polynomials including

$$
p_{3}=\left(m s_{12}-2 m+s_{12}-1\right)\left(m s_{12}+s_{12}-1\right)
$$

and

$$
\begin{aligned}
p_{4}= & -8 m s_{13}^{4}+8\left(m s_{12}+3 m-s_{12}+1\right) s_{13}^{3}-2\left(9 m s_{12}-4 s_{12}^{2}+14 m-s_{12}+5\right) s_{13}^{2} \\
& +2\left(7 m s_{12}-4 s_{12}^{2}+6 m+s_{12}+3\right) s_{13}-3 m s_{12}+2 s_{12}^{2}-2 m-s_{12}-1 .
\end{aligned}
$$

The roots of $p_{3}$ are $\sigma_{1}=\frac{2 m+1}{m+1}$ and $\sigma_{2}=\frac{1}{m+1}$. Substituting $\sigma_{1}$ into $p_{4}$, clearing denominators, and rescaling by a constant gives the value $p_{1}\left(s_{13}\right)$, which is nonzero for $m \in(-1,1)$ by the first statement in Lemma 6.1. Substituting $\sigma_{2}$ into $p_{4}$, clearing denominators, and rescaling by a constant gives the value $p_{2}\left(s_{13}\right)$. For the case $m \in(-1,1), p_{2}$ has four distinct positive real roots. Thus, in order to have a geometrically realizable solution, we 
must have $s_{12}=1 /(m+1)$ and $s_{13}$ must be one of the four positive roots of $p_{2}$. From equation (22), we also have that $s_{34}=(m+2) /(m+1)$.

Since $\lambda^{\prime}=-1$, we have $1 / \sqrt{-\lambda^{\prime}}=1$ in Propositions 2.2 and 2.4. If $m \in(0,1)$, then $s_{12}<1$ and $s_{34}>1$. Since we have saturated with respect to all possible differences of the remaining four $s_{i j}$ variables, the values of $s_{13}, s_{14}, s_{23}, s_{24}$ must all be distinct roots of $p_{2}$. By Lemma 6.1, two of these values are less than one, and two are greater than one. By Proposition 2.4 part 1., the configuration cannot be convex. Since we have saturated the Gröbner basis to eliminate symmetric solutions, it follows that for $m \in(0,1)$, the only strictly planar, asymmetric configurations are concave. By Proposition 2.2 part 1., vortices 3 and 4 (with equal strength $m$ ) lie on the outer triangle, while either vortex 1 or 2 can lie in the interior of the concave configuration. (The situation is reversed under the transformation $m \mapsto \frac{1}{m}$.)

For the special case $m=1$, we have that $s_{12}=3 / 2$ and $s_{34}=1 / 2$ or vice versa. If $s_{12}=3 / 2=\sigma_{1}$, then substitution into $p_{4}$ yields that $s_{13}$ is a root of $p_{1}$. But the only real roots of $p_{1}$ when $m=1$ are $1 / 2$ and $3 / 2$. Thus, we either have $s_{12}=s_{13}$ or $s_{34}=s_{13}$ and by equation (11), the configuration is necessarily a kite (a symmetric configuration). If $s_{12}=1 / 2=\sigma_{2}$, a similar argument with $p_{2}$ replacing $p_{1}$ also yields a kite configuration. This completes the proof of part 1 . of the theorem.

If $m \in(-1,0)$, we have $s_{12}>1, s_{34}>1$. Taken together with Lemma 6.1, this implies that four mutual distances are greater than one and two are less than one. Therefore, from Propositions 2.2 and 2.4, it follows that the only real, positive solutions to the system of equations given by $\tilde{G}_{I_{s}}$ correspond to convex asymmetric configurations where the equalstrength vortices are adjacent. This proves part 2 . of the theorem.

\subsection{Proof of Lemma 6.1}

In this section we analyze the polynomials $p_{1}$ and $p_{2}$ and prove Lemma 6.1. For $p_{1}$, we use Lemma 3.5, while for $p_{2}$, we make appropriate choices of Möbius transformations.

Both $p_{1}$ and $p_{2}$ are polynomials of degree four. Moreover, if $m>0$, their coefficients have four sign changes, and thus they have either four, two or zero positive real roots, according to Descartes' rule of signs. The roots of the polynomials can be obtained using Ferrari's formula for quartic equations [7]. However, we can understand a great deal about the solutions by using the resolvent cubic and Möbius transformations.

One important observation is that if we make the change $m \mapsto \frac{1}{m}$ in the polynomial $p_{1}$, and clear the denominators, we obtain $p_{2}$. In particular, this means that the roots of $p_{2}$ for an $m \in(0,1)$ are equivalent to the roots of $p_{1}$ for $m \in(1, \infty)$. Hence, it suffices to study the polynomials for $|m|<1$.

\section{Discriminants of $p_{1}$ and $p_{2}$}

The discriminant of $p_{1}$ is $256(m+1)^{4}(5 m+3)^{2}(2 m+1)^{2}(m-1)^{2}$, which is strictly positive except for the $m$-values $-1,-3 / 5,-1 / 2$ and 1 where it vanishes. At $m=-1, p_{1}$ reduces to a quadratic polynomial with repeated roots at $x=1 / 2$. For $m=-3 / 5, p_{1}$ has only 
complex roots, while for $m=-1 / 2, p_{1}$ has a double root at $x=0$. At $m=1$, there are double real roots at $x=1 / 2$ and $x=3 / 2$.

The discriminant for $p_{2}$ is $256(m+2)^{2}(3 m+5)^{2}(m+1)^{4} m^{2}(m-1)^{2}$ which is strictly positive except for the $m$-values $-2,-5 / 3,-1,0$ and 1 where it vanishes. Focusing on the values between -1 and 1 , we have that at $m=-1, p_{2}$ reduces to a quadratic polynomial with repeated roots at $x=1 / 2$. For $m=0, p_{2}$ has a double root at 1 and two other roots at $(3 \pm \sqrt{5}) / 2$. At $m=1, p_{2}$ has double real roots at $x=1 / 2$ and $x=3 / 2$. For all values of $m \in(-1,1)$, except for $m=0$, the four roots of $p_{2}$ are distinct.

\section{Polynomial $p_{1}$}

After applying the shift $x \mapsto x+(m+1)(5 m+3) /\left(4(m+1)^{2}\right)$ to remove the cubic term in $p_{1}$, we compute the key coefficients of the shifted quartic to be

$$
\begin{aligned}
p & =-\frac{11 m^{2}+6 m-1}{8(m+1)^{2}}, \quad \text { and } \\
p^{2}-4 r & =\frac{(m-1)(5 m+3)(7 m+3)}{16(m+1)^{3}} .
\end{aligned}
$$

It is straight-forward to check that either $p>0$ or $p^{2}-4 r<0$ (or both), for each $m$-value in $(-1,1)$. Using Lemma 3.5 , it follows that the roots of $p_{1}$ are all complex for $m \in(-1,1)$, except when $m=-1 / 2$, where 0 is a double root.

Polynomial $p_{2}$ : The case $m \in[0,1]$

Consider the intervals $J_{1}=\left[0, \frac{1}{2}\right], J_{2}=\left[\frac{1}{2}, 1\right], J_{3}=\left[1, \frac{3}{2}\right]$, and $J_{4}=\left[\frac{3}{2}, 5\right]$. We show that for each $m \in[0,1], p_{2}$ has a root in each of these intervals. The result follows from direct computation for $m=0$ and $m=1$, noting that some of the roots are a shared endpoint of adjacent intervals.

\begin{tabular}{l|ll}
\hline$x$-interval & Möbius Transformation \\
\hline$J_{1}$ & $x=\frac{1}{2} \frac{u}{u+1}, \quad m=\frac{\alpha}{\alpha+1}$ \\
$J_{2}$ & $x=\frac{u+\frac{1}{2}}{u+1}, \quad m=\frac{\alpha}{\alpha+1}$ \\
$J_{3}$ & $x=\frac{3}{2} u+1$ \\
$J_{4}$ & $x=\frac{5 u+\frac{3}{2}}{u+1}$, & $m=\frac{\alpha}{\alpha+1}$ \\
&
\end{tabular}


Using the Möbius transformations given in the table above, after clearing the denominators, we obtain the following four polynomials

$$
\begin{aligned}
P_{J_{1}}= & -(2 \alpha+1) u^{4}-2(2 \alpha+1) u^{3}+2\left(8 \alpha^{2}+11 \alpha+4\right) u^{2}+4\left(12 \alpha^{2}+17 \alpha+6\right) u+36 \alpha^{2}+48 \alpha+16 \\
P_{J_{2}}= & 4 \alpha^{2} u^{4}+4\left(4 \alpha^{2}+\alpha\right) u^{3}+2\left(8 \alpha^{2}-\alpha-2\right) u^{2}-6(2 \alpha+1) u-2 \alpha-1 \\
P_{J_{3}}= & -(18 \alpha+5) u^{4}-2(22 \alpha+5) u^{3}+2\left(8 \alpha^{2}-13 \alpha-2\right) u^{2}+4 \alpha(4 \alpha-1) u+4 \alpha^{2} \\
P_{J_{4}}= & 4\left(3969 \alpha^{2}+3352 \alpha+704\right) u^{4}+4\left(1764 \alpha^{2}+815 \alpha+16\right) u^{3}+2\left(392 \alpha^{2}-397 \alpha-218\right) u^{2} \\
& -2(134 \alpha+45) u-18 \alpha-5 .
\end{aligned}
$$

Using Descartes' rule of signs, it is straight-forward to show that for any $\alpha>0$, each of the polynomials above has precisely one positive real root. The values at the endpoints of each $J_{i}$ can be determined by direct substitution. It follows that $p_{2}=0$ has one solution in each of the intervals $J_{1}, J_{2}, J_{3}$ and $J_{4}$.

Polynomial $p_{2}$ : The case $m \in(-1,0)$

Consider the intervals $K_{1}=\left[0, \frac{1}{2}\right], K_{2}=\left[\frac{1}{2}, 1\right], K_{3}=\left[1, \frac{m+2}{m+1}\right]$, and $K_{4}=\left[\frac{m+2}{m+1}, \infty\right]$. We show that for each $m \in(-1,0), p_{2}$ has a root in each of these intervals.

$$
\begin{aligned}
& \begin{array}{l|ll}
\hline x \text {-interval } & \multicolumn{1}{|c}{\text { Möbius Transformation }} \\
\hline K_{1} & x=\frac{1}{2} \frac{u}{u+1}, & m=-\frac{1}{\alpha+1} \\
K_{2} & x=\frac{u+\frac{1}{2}}{u+1}, & m=-\frac{1}{\alpha+1} \\
K_{3} & x=\frac{1+\frac{m+2}{m+1} u}{u+1}, & m=-\frac{1}{\alpha+1} \\
K_{4} & x=u+\frac{m+2}{m+1}, & m=-\frac{1}{\alpha+1}
\end{array} \\
& P_{K_{1}}=\left(\alpha^{2}+2 \alpha\right) u^{4}+2\left(\alpha^{2}+2 \alpha\right) u^{3}-2\left(4 \alpha^{2}+5 \alpha+2\right) u^{2} \\
& -2\left(12 \alpha^{2}+14 \alpha+4\right) u-4\left(4 \alpha^{2}+4 \alpha+1\right) \\
& P_{K_{2}}=-4 u^{4}+4(\alpha-2) u^{3}+2\left(2 \alpha^{2}+7 \alpha-2\right) u^{2}+6\left(\alpha^{2}+2 \alpha\right) u+\left(\alpha^{2}+2 \alpha\right) \\
& P_{K_{3}}=4\left(4 \alpha^{4}+12 \alpha^{3}+9 \alpha^{2}+2 \alpha\right) u^{4}+8\left(6 \alpha^{4}+17 \alpha^{3}+11 \alpha^{2}+2 \alpha\right) u^{3} \\
& +8\left(2 \alpha^{4}+2 \alpha^{3}-10 \alpha^{2}-9 \alpha-2\right) u^{2}-8\left(\alpha^{3}+5 \alpha+2\right) u-4 \alpha^{2} \\
& P_{K_{4}}=-16 \alpha^{3} u^{4}-8\left(6 \alpha^{3}+4 \alpha^{2}\right) u^{3}-8\left(4 \alpha^{3}+5 \alpha^{2}+2 \alpha\right) u^{2} \\
& +8\left(2 \alpha^{3}+5 \alpha^{2}+2 \alpha\right) u+4\left(4 \alpha^{3}+12 \alpha^{2}+9 \alpha+2\right) \text {. }
\end{aligned}
$$

Using Descartes' rule of signs, it is straight-forward to show that for any $\alpha>0$, each of the polynomials above has one positive real root. Again, it is traightforward to check the behavior at the endpoints of each interval by direct substitution. It follows that $p_{2}=0$ has one solution in each of the intervals $K_{1}, K_{2}, K_{3}$ and $K_{4}$. 


\subsection{The variety of asymmetric configurations}

In this section we study the asymmetric solutions to our problem and show that there are exactly eight asymmetric solutions for each $m \in(-1,1)$. We restrict to the case $\lambda^{\prime}=-1$ since $\lambda^{\prime}>0$ only leads to symmetric solutions (as shown in Section 7.3.) We first recall some definitions and theorems from algebraic geometry (see [6] and [11] for more details).

Definition 6.3. Let $A$ be a commutative ring.

1. An ideal $I$ of $A$ is said to be real if, for every sequence $a_{1}, \ldots, a_{p}$ of elements of $A$, we have

$$
a_{1}^{2}+\ldots a_{p}^{2} \in I \Longrightarrow a_{i} \in I, \text { for } i=1, \ldots, p
$$

2. $\sqrt[R]{I}$ is the smallest real ideal of $A$ containing $I$ and is called the real radical of the ideal $I$.

Let $k$ be a field. If $I \subset k\left[x_{1}, \ldots, x_{n}\right]$ is an ideal, we denote by $\mathbf{V}(I)$ the set

$$
\mathbf{V}(I)=\left\{\left(a_{1}, \ldots, a_{n}\right) \in k^{n}: f\left(a_{1}, \ldots, a_{n}\right)=0 \text { for all } f \in I\right\} .
$$

$\mathbf{V}(I)$ is an affine variety. In particular if $I=<f_{1}, \ldots, f_{s}>$, then $\mathbf{V}(I)=\mathbf{V}\left(f_{1}, \ldots, f_{s}\right)$. If $k$ is the field of the real numbers $\mathbb{R}$ we will say $\mathbf{V}(I)$ is a real algebraic variety. Note that while this terminology is common in algebraic geometry books, it is different from the terminology frequently used in real algebraic geometry (see [6], for example).

Definition 6.4. Let $V \subset k^{n}$ be an affine variety. Then we set

$$
\mathbf{I}(V)=\left\{f \in k\left[x_{1}, \ldots, x_{n}\right]: f\left(a_{1}, \ldots a_{n}\right)=0 \text { for all }\left(a_{1}, \ldots a_{n}\right) \in V\right\}
$$

$\mathbf{I}(V)$ is an ideal and it is called the ideal of the variety $V$.

We are now ready to state a version of the Real Nullstellensatz:

Theorem 6.5 (Real Nullstellensatz). Let $k$ be a real closed field and $I$ an ideal of $k\left[x_{1}, \ldots, x_{n}\right]$. Then $\mathbf{I}(\mathbf{V}(I))=\sqrt[R]{I}$.

The dimension of an affine variety $V \subset k^{n}$, denoted $\operatorname{dim} V$, is the degree of the affine Hilbert polynomial of the corresponding ideal $\mathbf{I}(V) \subset k\left[x_{1}, \ldots, x_{n}\right]$ (see [11 for more details). This degree can be easily computed using Singular. In the case $k=\mathbb{R}$ one needs to know the ideal of the variety, or by the Real Nullstellensatz, the real radical of the ideal. The real radical can be computed using the realrad.lib [26] library of SingulaR [12]. More details about the algorithms can be found in the paper [25]. We now apply the above theory to our problem.

Theorem 6.6. Consider the ring $\mathbb{R}\left[s_{12}, s_{13}, s_{14}, s_{23}, s_{24}, s_{34}, m\right]$. The asymmetric relative equilibria configurations form a one-dimensional real variety $V \subset \mathbb{R}^{7}$. 
Proof. Consider the Gröbner basis $G_{I_{s}}$ for the ideal $I_{s}$ and saturate with respect to $s_{13}, s_{14}, s_{24},\left(s_{13}-s_{24}\right),\left(s_{14}-s_{23}\right),\left(s_{13}-s_{14}\right),\left(s_{23}-s_{24}\right),\left(s_{13}-s_{23}\right)$, and $\left(s_{14}-s_{24}\right)$, as before. In addition, saturate with respect to $\left(m s_{12}-2 m+s_{12}-1\right)$ to eliminate the case $s_{12}=(2 m+1) /(m+1)$ for which the solutions are complex. We obtain the following polynomials:

$$
\begin{aligned}
& f_{1}=s_{13}+s_{14}+s_{23}+s_{24}-2 s_{34}-1 \\
& f_{2}=s_{12}-s_{34}+1 \\
& f_{3}=s_{34} m+s_{34}-m-2 \\
& f_{4}=2 s_{24}^{2}-s_{14} s_{34}-s_{23} s_{34}-4 s_{24} s_{34}+2 s_{34}^{2}+2 s_{23} \\
& f_{5}=2 s_{23} s_{24}-2 s_{23}-2 s_{24}+s_{34} \\
& f_{6}=2 s_{14} s_{24}-s_{34} \\
& f_{7}=2 s_{23}^{2}+s_{14} s_{34}-3 s_{23} s_{34}+2 s_{24}-s_{34} \\
& f_{8}=2 s_{14} s_{23}-s_{14} s_{34}-s_{23} s_{34}+s_{34} \\
& f_{9}=2 s_{14}^{2}-3 s_{14} s_{34}+s_{23} s_{34}-2 s_{14}-2 s_{23}-2 s_{24}+3 s_{34}+2 .
\end{aligned}
$$

Let $I=<f_{1}, \ldots, f_{9}>$. We want to find the dimension of the real variety $\mathbf{V}(I)$. First, we find $\mathbf{I}(\mathbf{V}(I))$ or $\sqrt[R]{I}$. This can be computed using the realrad.lib [26] library of Singular [12]. However in this case it turns out that $\sqrt[R]{I}=I$, and hence $f_{1}, \ldots, f_{9}$ are the generators of the real radical of $I$. The degree of the affine Hilbert polynomial of $\sqrt[R]{I}=I$ is one, and thus $\mathbf{V}(I)$ is a one dimensional real algebraic variety.

We now want to see if the variety contains singular points. First we recall some definitions and theorems.

Definition 6.7. Let $V \subset k^{n}$ be an affine algebraic variety then the Zariski tangent space of $V$ at $p=\left(p_{1}, \ldots, p_{n}\right)$ denoted by $T_{p}(V)$ is the variety

$$
T_{p}(V)=\mathbf{V}\left(d_{p}(f): f \in \mathbf{I}(V)\right)
$$

where $d_{p}(f)=\sum_{i=1}^{n} \frac{\partial f}{\partial x_{i}}(p)\left(x_{i}-p_{i}\right)$.

Clearly if $\mathbf{I}(V)=<f_{1}, \ldots, f_{s}>$ then $T_{p}(V)=\mathbf{V}\left(d_{p}\left(f_{1}\right), \ldots, d_{p}\left(f_{s}\right)\right)$, and it is the translate of a linear subspace of $k^{n}$. Recall that if $V \subset k^{n}$ is an affine variety, then $V$ is irreducible if and only if $\mathbf{I}(V)$ is a prime ideal. Thus we have the following definition:

Definition 6.8. Let $V \subset k^{n}$ be an irreducible variety. A point $p$ in $V$ is nonsingular (or smooth) provided that $\operatorname{dim} T_{p}(V)=\operatorname{dim} V$. In other words, if $\mathbf{I}(V)=<f_{1}, \ldots, f_{s}>$, then $p$ is nonsingular if and only if the rank of the Jacobian matrix $J=\left[\frac{\partial f_{i}}{\partial x_{j}}\right]$ is equal to $n-\operatorname{dim}(V)$. Otherwise, $p$ is a singular point of $V$.

The notion of Zariski tangent space is defined even at a singular point. However, when $V \subset \mathbb{R}^{n}$ is an irreducible variety and $p \in V$ is a nonsingular point, a neighbourhood of $p$ in $V$ is a $C^{\infty}$ submanifold of $\mathbb{R}^{n}$ and the tangent space of $V$ at $z$ (in the $C^{\infty}$ sense) coincide with the Zariski tangent space. If $p$ is singular, then the dimension of $T_{p}(V)$ is bigger than the dimension of $V$. 
Theorem 6.9. The variety of the asymmetric strictly planar relative equilibria configurations has no singular points and hence it is a smooth one-dimensional manifold.

Proof. The Jacobian matrix of $\left(f_{1}, \ldots, f_{9}\right)$ is

$\left[\begin{array}{ccccccc}0 & 1 & 1 & 1 & 1 & -2 & 0 \\ 1 & 0 & 0 & 0 & 0 & -1 & 0 \\ 0 & 0 & 0 & 0 & 0 & m+1 & s_{34}-1 \\ 0 & 0 & -s_{34} & -s_{34}+2 & 4\left(s_{24}-s_{34}\right) & -s_{14}-s_{23}-4\left(s_{24}-s_{34}\right) & 0 \\ 0 & 0 & 0 & 2 s_{24}-2 & 2 s_{23}-2 & 1 & 0 \\ 0 & 0 & 2 s_{24} & 0 & 2 s_{14} & -1 & 0 \\ 0 & 0 & s_{34} & 4 s_{23}-3 s_{34} & 2 & s_{14}-3 s_{23}-1 & 0 \\ 0 & 0 & 2 s_{23}-s_{34} & 2 s_{14}-s_{34} & 0 & -s_{14}-s_{23}+1 & 0 \\ 0 & 0 & 4 s_{14}-3 s_{34}-2 & s_{34}-2 & -2 & -3 s_{14}+s_{23}+3 & 0\end{array}\right]$

Using Gaussian elimination we obtain the following matrix

$\left[\begin{array}{cccc:ccc}1 & 0 & 0 & 0 & 0 & -1 & 0 \\ 0 & 1 & 1 & 1 & 1 & -2 & 0 \\ 0 & 0 & s_{34} & 4 s_{23}-3 s_{34} & 2 & s_{14}-3 s_{23}-1 & 0 \\ 0 & 0 & 0 & 2 s_{24}-2 & 2 s_{23}-2 & 1 & 0 \\ \hdashline 0 & 0 & 0 & 0 & & \end{array}\right]$

where

$$
A=\left[\begin{array}{ccc}
q_{1} & q_{2} & 0 \\
0 & m+1 & s_{34}-2 \\
0 & a & 0 \\
0 & b & 0 \\
0 & c & 0
\end{array}\right]
$$

and $a, b, c$ are polynomials that belong to the ideal of the variety (and hence vanish on the variety), and

$$
\begin{aligned}
& q_{1}=-4\left(s_{23}-s_{24}\right)\left(2 s_{23}+2 s_{24}-2 s_{34}-1\right) \\
& q_{2}=-8 s_{23} s_{24}-8 s_{24}{ }^{2}+8 s_{24} s_{34}+6 s_{24}+4 s_{23}-4 s_{34} .
\end{aligned}
$$

Since $s_{34} \neq 0, s_{24}-1 \neq 0, q_{1} \neq 0$, and $m_{3}-1$ and $s_{34}-2$ are not both zero on the variety, it follows that the rank of the Jacobian matrix is 6 . Since $\operatorname{dim}(V)=1$, by Definition 6.8 the variety has no singular points. It follows that it is a smooth manifold.

Theorem 6.10. For each value of $m$ in $(-1,1)$, there are exactly four (eight counting reflected solutions) strictly planar asymmetric relative equilibria configurations. They are convex if $m<0$ and concave if $m>0$. 


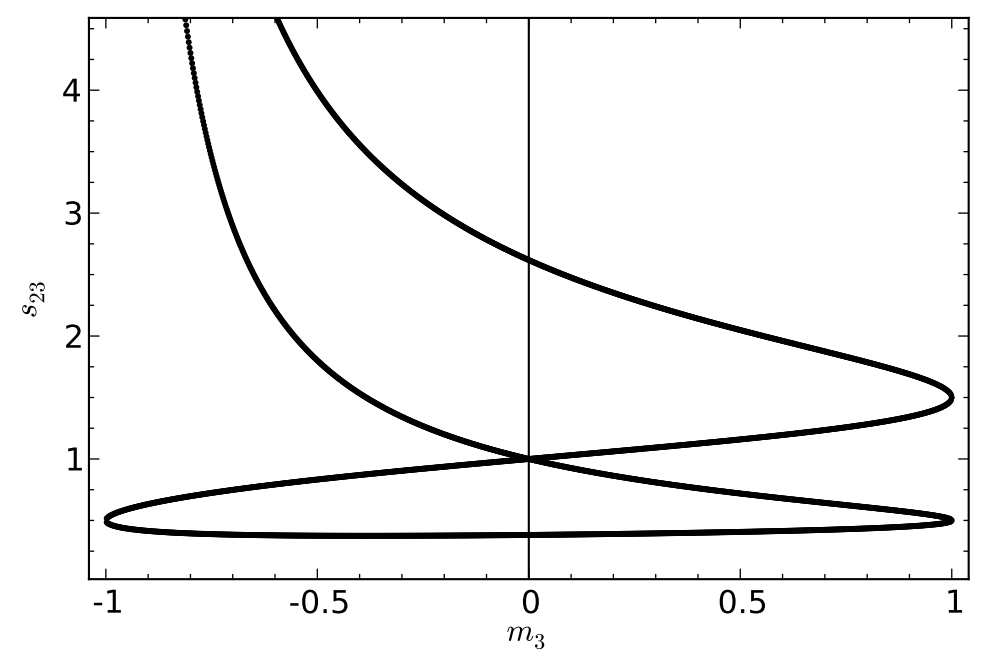

Figure 2: Projection of part of the manifold of asymmetric configurations onto the plane $\left(m, s_{23}\right)$. As $m \rightarrow-1, s_{23} \rightarrow \infty$ along two branches while $s_{23} \rightarrow \frac{1}{2}$ along the other two branches.

Proof. The proof consists of showing that the system of equations $f_{1}=0, \ldots, f_{9}=0$ has four solutions for each value of $m$ in $(-1,1)$. The convexity of the configuration then follows from Theorem 6.2. We use an algorithm for the triangular decomposition of semialgebraic systems. Such an algorithm, given a system of equations and inequalities $S$, computes simpler systems $S_{1}, \ldots, S_{k}$ such that a point is a solution of the original system $S$ if and only if it is a solution of one of the systems $S_{1}, \ldots, S_{k}$. Each of these systems has a triangular shape and remarkable properties: for this reason it is called a regular semialgebraic system and the set of the $S_{1}, \ldots, S_{k}$ is called a full triangular decomposition of $S$. See [9] and references therein for some background. The algorithm is detailed in [9] and it is available in Maple15 $5^{\mathrm{TM}}$ via the RealTriangularize command of the RegularChains package. A full triangular decomposition of the system $f_{1}=0, \ldots, f_{9}=0$ is given below.

If $m=0$ we have the following two systems:

$$
\begin{array}{ll}
s_{12}-1=0 & s_{12}-1=0 \\
s_{13}+s_{23}-3=0 & s_{13}-1=0 \\
s_{14}-1=0 & s_{14}+s_{24}-3=0 \\
s_{23}^{2}-3 s_{23}+1=0 & s_{23}-1=0 \\
s_{24}-1=0 & s_{34}^{2}-3 s_{24}+1=0 \\
s_{34}-2=0 & s_{34}-2,
\end{array}
$$


if $m=1$ we have:

$$
\begin{array}{ll}
2 s_{12}-1=0 & 2 s_{12}-1=0 \\
2 s_{13}-1=0 & 2 s_{13}-3=0 \\
2 s_{14}-1=0 & 2 s_{14}-3=0 \\
2 s_{23}-3=0 & 2 s_{23}-1=0 \\
2 s_{24}-3=0 & 2 s_{24}-1=0 \\
2 s_{34}-3=0 & 2 s_{34}-3=0
\end{array}
$$

and if $m \in(-1,0) \cup(0,1)$ we obtain:

$$
\begin{aligned}
& s_{12}-s_{34}+1=0 \\
& s_{13}+s_{14}+s_{23}+s_{24}-2 s_{34}-1=0 \\
& s_{34} s_{14}+\left(s_{34}-2\right) s_{23}-2 s_{24}^{2}+4 s_{24} s_{34}-2 s_{34}^{2}=0 \\
& \left(2 s_{24}-2\right) s_{23}-2 s_{24}+s_{34}=0 \\
& 4 s_{24}^{4}+\left(-8 s_{34}-4\right) s_{24}^{3}+\left(4 s_{34}^{2}+6 s_{34}+4\right) s_{24}^{2}+\left(-4 s_{34}^{2}-2 s_{34}\right) s_{24}+s_{34}^{2}=0 \\
& (m+1) s_{34}-m-2=0
\end{aligned}
$$

This decomposition immediately shows that the system has no real solutions if $m=-1$, two real solutions if $m=1$, and four real solutions if $m=0$. It remains to study the case $m \in(-1,0) \cup(0,1)$. Solving the last equation of the last system of the triangular decomposition yields $s_{34}=\frac{m+2}{m+1}$. Substituting this into the preceding equation of the same system, after clearing the denominators, yields $p_{2}\left(s_{24}\right)$. From Lemma 6.1 we know that $p_{2}$ has four distinct positive real roots for $m \in(-1,0) \cup(0,1)$. If we fix one such solution for $s_{24}$ and substitute into the first four equations of the system, the resulting system is $A x=b$, where $x=\left(s_{12}, s_{13}, s_{14}, s_{23}\right)$,

$$
A=\left[\begin{array}{cccc}
1 & 0 & 0 & 0 \\
0 & 1 & 1 & 1 \\
0 & 0 & s_{34} & \left(s_{34}-2\right) \\
0 & 0 & 0 & 2\left(s_{24}-1\right)
\end{array}\right]
$$

and $b=\left[-1+s_{34},-s_{24}+2 s_{34}+1, s_{24}^{2}-4 s_{24} s_{34}+2 s_{34}^{2}, 2 s_{24}-s_{34}\right]^{T}$. Since the matrix $A$ is invertible, $A x=b$ has exactly one solution for every vector $b$. This proves the theorem since it implies that the system $f_{1}=0, \ldots f_{9}=0$ has four real positive solutions.

Remark. Note that for $m=1$ we obtain two solutions which correspond to an equilateral triangle configuration with a vortex at the center. These are symmetric configurations that have not been eliminated because in the case of equal vorticities, there is an additional symmetry that we did not exclude when saturating. Also, note that the polynomials $f_{1}, \ldots, f_{9}$ were obtained saturating with respect to $\left(m s_{12}-2 m+s_{12}-1\right)$. This eliminated 
the real solutions of the system $f_{1}=0, \ldots, f_{9}=0$ for $m \in(-\infty,-1) \cup(1, \infty)$. However, we know that the number of real (positive solutions) of the original system (e.g., before saturating) for $m \in(-\infty,-1) \cup(1, \infty)$ is the same as the number of solutions of $f_{1}=$ $0, \ldots, f_{9}=0$ for $m \in(-1,1)$.

\section{Symmetric Strictly Planar Relative Equilibria}

In this section we investigate all possible symmetric, strictly planar, relative equilibria in the four-vortex problem with $\Gamma_{1}=\Gamma_{2}=1$ and $\Gamma_{3}=\Gamma_{4}=m$. The four possible configurations are an isosceles trapezoid, a concave kite, a convex kite, or a rhombus. We separate the kite configurations into two cases based on the sign of $\lambda^{\prime}$.

\subsection{The isosceles trapezoid family}

Without loss of generality, suppose that the vortices are ordered sequentially around an isosceles trapezoid, so that the lengths of the diagonals are given by $r_{13}=r_{24}$ and the congruent legs have length $r_{14}=r_{23}$. Due to the symmetry of the configuration, the four vortices lie on a common circle, that is, the isosceles trapezoid is a cyclic quadrilateral. By Ptolemy's theorem, we have that

$$
r_{13}^{2}=r_{24}^{2}=r_{12} r_{34}+r_{14}^{2}
$$

A choice of six mutual distances satisfying $r_{13}=r_{24}, r_{14}=r_{23}$ and equation (24) will also satisfy $e_{C M}=0$.

In contrast to the Newtonian four-body problem, the isosceles trapezoid family of four-vortex relative equilibria can be solved completely (by hand) in terms of the vortex strength $m$ (compare with [10]). Let $x=r_{34} / r_{12}$ and $y=r_{14} / r_{12}$. Rather than assuming a specific scaling on the distances (e.g., $\lambda^{\prime}=-1$ ), we will solve for $x$ and $y$ in terms of $m$. Using the symmetries of the configuration and equation (24), equation (10) reduces to

$$
2\left(y^{2}-x^{2}\right)\left(y^{2}-1\right)+x\left(2 y^{2}-x^{2}-1\right)=0 .
$$

This relation between $x$ and $y$ is both necessary and sufficient for the trapezoid to be a relative equilibrium.

Focusing on the first and last columns of equations (9), the first and last equations are easily satisfied due to symmetry. The middle four equations in (9) are equivalent given that (25) holds. They determine a formula for $m$ given by

$$
m=\frac{x\left(1-y^{2}\right)}{y^{2}-x^{2}}
$$

where we have used $\left|A_{i}\right|=r_{j k} r_{j l} r_{k l} /\left(4 r_{c}\right)$ for the area of a triangle circumscribed in a circle of radius $r_{c}$. Solving equation (26) for $y^{2}$ and substituting into equation (25) leads to the equation

$$
x(1-x)(1+x)\left(x^{2}(2 m+1)-m(m+2)\right)=0 .
$$


Since $x$ represents the ratio of two distances, there are only two possibilities. If $x=1$, then equation (25) quickly gives $y=1$ and the configuration is a square. The second equation in (9) then gives that $m=1$ and all vortices must have the same strength. The other possibility is that $x^{2}=m(m+2) /(2 m+1)$. Substituting this value into equation (26) and solving for $y^{2}$ yields

$$
y^{2}=\frac{1}{2}\left(m+2-\sqrt{\frac{m(m+2)}{2 m+1}}\right) \text {. }
$$

Note that the necessary condition $x^{2}>0$ is satisfied only for $m>0$ and $-2<m<-1 / 2$. In order for the expression for $y^{2}$ to be real, we must be in one of these two regimes. However, while $y^{2}>0$ holds for $m>0$, it is not satisfied on $-2<m<-1 / 2$. Therefore, there does not exist an isosceles trapezoid solution for the case $m<0$.

Theorem 7.1. There exists a one-parameter family of isosceles trapezoid relative equilibria with vortex strengths $\Gamma_{1}=\Gamma_{2}=1$ and $\Gamma_{3}=\Gamma_{4}=m$. The vortices 1 and 2 lie on one base of the trapezoid, while 3 and 4 lie on the other. Let $\alpha=m(m+2) /(2 m+1)$. If $r_{13}=r_{24}$ are the lengths of the two congruent diagonals, then the mutual distances are described by

$$
\begin{gathered}
\left(\frac{r_{34}}{r_{12}}\right)^{2}=\alpha, \quad\left(\frac{r_{14}}{r_{12}}\right)^{2}=\frac{1}{2}(m+2-\sqrt{\alpha}) \\
\text { and }\left(\frac{r_{13}}{r_{12}}\right)^{2}=\frac{1}{2}(m+2+\sqrt{\alpha}) .
\end{gathered}
$$

This family exists if and only if $m>0$. The case $m=1$ reduces to the square. For $m \neq 1$, the larger pair of vortices lie on the longest base.

Proof. The formula for $r_{13}^{2} / r_{12}^{2}$ comes from equation (24). Substituting $m=1$ into the formulas above quickly gives $r_{12}=r_{14}=r_{34}=r_{23}$ and $r_{13}=r_{24}=\sqrt{2} r_{12}$, a square. The only other item that remains to be shown is the fact about the larger vortices lying on the longer base. This follows since $\alpha>1$ if and only if $m>1$.

Remark. 1. If $0<m<1, r_{12}$ is the longest base length and the formulas in the theorem give $r_{34}<r_{14}<r_{12}<r_{13}$. On the other hand, if $m>1$, then $r_{34}$ is the longest base length and we deduce $r_{12}<r_{14}<r_{34}<r_{13}$. Both cases agree with the conclusion of statement 1 in Proposition 2.4.

2. The case $m>1$ is identical to the case $0<m<1$ through a rescaling of the vortex strengths and interchanging bodies 1 and 3, and bodies 2 and 4. Specifically, replacing $m$ by $1 / m$ and interchanging distances $r_{12}$ and $r_{34}$ leaves the three equations for the ratios of mutual distances unchanged. The vortex strengths map to $\Gamma_{1}=\Gamma_{2}=1$ and $\Gamma_{3}=\Gamma_{4}=1 / m<1$ under this transformation.

3. As $m \rightarrow 0, \alpha \rightarrow 0$ and consequently $r_{34} \rightarrow 0$. The limiting configuration is an equilateral triangle with vortices 3 and 4 colliding. As $m$ increases, so does $\alpha$, and the smaller base 
length $r_{34}$ approaches the larger one $r_{12}$ as $m \rightarrow 1$. The ratio of the diagonal to the larger base also increases monotonically with $m$. However, the ratio of the leg to the larger base begins at $1(m=0)$ and decreases to a minimum value of approximately 0.904781 before increasing back to 1 at the square configuration. This minimum value occurs at $m \approx 0.234658$, which is the only positive root of the quartic $8 m^{4}+19 m^{3}+9 m^{2}+m-1$. Just as with the Newtonian case (see [10]), the range of $y$ is surprisingly small, confined to the interval $[0.904781,1]$.

\subsection{Kite configurations: $\lambda^{\prime}<0$}

In this section, we consider kite central configurations when $\lambda^{\prime}<0$. Such configurations contain two vortices that are symmetrically located with respect to an axis of symmetry while the remaining two vortices lie on the axis of symmetry. The configuration formed by the vortices can either be concave or convex. In this section, we focus on configurations with only one line of symmetry. Those configurations with two lines of symmetry, i.e., the rhombus configurations, are studied in detail in Section 7.4.

Our goal is to count the number of kite configurations as $m$ varies and describe the type of possible configurations. Recall that $\widetilde{\mathcal{F}}, \widetilde{\mathcal{G}}, \tilde{e}_{C M}$ and $\widetilde{\mathcal{H}}$ represent the Albouy-Chenciner equations, the unsymmetrized Albouy-Chenciner equations, the Cayley-Menger determinant and the Dziobek equations, respectively, in terms of the variables $s_{i j}=r_{i j}^{2}$, with $\lambda^{\prime}=-1$. Denote the complete system of these equations as $\widetilde{\mathcal{E}}$. Our goal is to prove the following theorem.

Theorem 7.2. For each value of $m \in(-\infty,-2) \cup\left(-\frac{1}{2}, 0\right) \cup(0, \infty)$, system $\widetilde{\mathcal{E}}$ has exactly four (real, positive) solutions that correspond to symmetric kite configurations. If $m \in(0, \infty)$, such configurations are concave. If $m \in\left(-\frac{1}{2}, 0\right)$, then there are two convex configurations with $\Gamma_{3}$ and $\Gamma_{4}$ on the axis of symmetry, and two concave ones with $\Gamma_{1}$ and $\Gamma_{2}$ on the axis of symmetry. If $m \in(-\infty,-2)$, the situation is reversed and there are two concave configurations with $\Gamma_{3}$ and $\Gamma_{4}$ on the axis of symmetry, and two convex ones with $\Gamma_{1}$ and $\Gamma_{2}$ on the axis of symmetry. There are no other strictly kite configurations with $\lambda^{\prime}<0$ for other values of $m$.

Remark. 1. As we show below, the case $m=1$ is considerably different from the Newtonian four-body problem. In fact, it is known for the Newtonian case that there are two distinct symmetric kite configurations for four equal masses: an equilateral triangle with a mass in the center and an isosceles triangle with a mass on the axis of symmetry [1]. Instead, for four vortices of equal strength, the only concave kite central configuration is an equilateral triangle with a vortex at the center of the triangle.

2. Based on this theorem and Theorem 6.10, we conclude that there are no concave relative equilibria when $m \in(-1,-1 / 2)$. This is another contrast with the Newtonian fourbody problem, where it is shown in 14 that for any choice of four positive masses, there exits a concave central configuration. 
3. The exact count on the number of concave symmetric solutions given in Table 1 is precisely twice the values expressed in the theorem. This is due to the fact that each solution found can be reflected about the axis of symmetry, a transformation that does not change the mutual distances $r_{i j}$, but does alter the positions $x_{i}$.

We have two possibilities, either vortices 3 and 4 lie on the axis of symmetry or vortices 1 and 2 do. It is convenient to study the two cases separately, since the conditions imposed by the symmetry are different. The first case will be explored in Lemma 7.3 , and the second in Lemma 7.4. The proof of Theorem 7.2 follows immediately from the two lemmas.

Let us consider the first case. If vortices 3 and 4 lie on the axis of symmetry, then $s_{23}=s_{13}$ and $s_{24}=s_{14}$. We compute a Gröbner basis for the ideal $\left\langle\overline{\mathcal{F}}, \overline{\mathcal{G}}, \bar{e}_{C M}, \bar{H}\right\rangle$, where $\overline{\mathcal{F}}, \overline{\mathcal{G}}, \bar{e}_{C M}, \overline{\mathcal{H}}$ are obtained from $\widetilde{\mathcal{F}}, \widetilde{\mathcal{G}}, \widetilde{e}_{C M}$ and $\widetilde{\mathcal{H}}$, respectively, by imposing the conditions $s_{23}=s_{13}$ and $s_{24}=s_{14}$. Then we saturate with respect to $s_{13}-s_{14}$, in order to exclude the rhombus configurations. We also saturate with respect to $s_{12}, s_{13}, s_{14}, s_{34}$ and $m$. This computation yields the following ten polynomials:

$$
\begin{aligned}
g_{1}= & s_{34} m+s_{13}+s_{14}+s_{34}-m-2 \\
g_{2}= & s_{13} m+s_{14} m+s_{12}+s_{13}+s_{14}+s_{34}-3 m-3 \\
g_{3}= & 2 s_{12} s_{34}-2 s_{13} s_{34}-2 s_{14} s_{34}+2 s_{34}^{2}-s_{12}+2 s_{13}+2 s_{14}+s_{34}-3 \\
g_{4}= & 2 s_{13} s_{14}-s_{13}-s_{14}+s_{34} \\
g_{5}= & 2 s_{13}^{2}+2 s_{14}^{2}-2 s_{13} s_{34}-2 s_{14} s_{34}+s_{12}-4 s_{13}-4 s_{14}+s_{34}+3 \\
g_{6}= & 2 s_{12} s_{13}+2 s_{12} s_{14}-s_{12} m-2 s_{12}-s_{13}-s_{14}+s_{34} \\
g_{7}= & s_{12} m^{2}+2 s_{12}^{2}+2 s_{13} s_{34}+2 s_{14} s_{34}-2 s_{34}^{2}-3 s_{12} m-4 s_{12} \\
& -s_{13}-s_{14}-2 s_{34}+2 m+4 \\
g_{8}= & 2 s_{14}^{2} m+2 s_{12} s_{14}+2 s_{14}^{2}+2 s_{14} s_{34}-6 s_{14} m-s_{12}+s_{13}-5 s_{14}-s_{34} \\
& +2 m+1 \\
g_{9}= & 4 s_{14}^{3}-4 s_{14}^{2} s_{34}+2 s_{12} s_{14}-10 s_{14}^{2}-2 s_{13} s_{34}+2 s_{14} s_{34}+2 s_{34}^{2}-s_{12} \\
& +s_{13}+7 s_{14}+2 s_{34}-3 \\
g_{10}= & 4 s_{12} s_{14}^{2}-2 s_{12} s_{14} m-4 s_{12} s_{14}-2 s_{14}^{2}-2 s_{13} s_{34}+2 s_{34}^{2}+s_{12} m+s_{12} \\
& +2 s_{13}+2 s_{14}+s_{34}-3 .
\end{aligned}
$$

Let us denote the corresponding system of equations $g_{1}=0, \ldots, g_{10}=0$ as $G$. Then the positive solutions of $G$ give the symmetric kite configurations described above. An exact count of these solutions is described in the following lemma.

Lemma 7.3. For each value of $m \in(0,1)$, system $G$ has exactly four real positive solutions corresponding to concave configurations. For each value of $m \in(-\infty,-2) \cup\left(-\frac{1}{2}, 0\right)$, system $G$ has exactly two real positive solutions. Such solutions correspond to convex configurations if $m \in\left(-\frac{1}{2}, 0\right)$ and to concave configurations if $m \in(-\infty,-2)$. For $m=1$, system $G$ has exactly two real positive solutions corresponding to an equilateral triangle with either vortex 3 or 4 at the center. There are no positive real solutions for other values of $m$. 
Proof. Computing a full triangular decomposition of system $G$ with the positivity conditions $s_{12}>0, s_{13}>0, s_{14}>0$ and $s_{34}>0$, we obtain five simpler systems. If $m=1$, we have the following two systems:

$$
T_{1}=\left\{\begin{array}{l}
2 s_{12}-3=0 \\
2 s_{13}-3=0 \\
2 s_{14}-1=0 \\
2 s_{34}-1=0,
\end{array} \quad T_{2}=\left\{\begin{array}{l}
2 s_{12}-3=0 \\
2 s_{13}-1=0 \\
2 s_{14}-3=0 \\
2 s_{34}-1=0
\end{array}\right.\right.
$$

If $m=0$, we have the following two systems:

$$
T_{3}=\left\{\begin{array}{c}
s_{12}-1=0 \\
s_{13}-1=0 \\
4 s_{14}-1=0 \\
4 s_{34}-3=0
\end{array} \quad T_{4}=\left\{\begin{array}{c}
s_{12}-1=0 \\
4 s_{13}-2=0 \\
s_{14}-1=0 \\
4 s_{34}-3=0
\end{array}\right.\right.
$$

If $m \in(-\infty,-2) \cup(-1 / 2,0) \cup(0,1)$, we obtain the system

$$
T_{5}=\left\{\begin{array}{l}
s_{12}+\left(-m^{2}-2 m\right) s_{34}-1+m^{2}=0 \\
s_{13}+s_{14}+(m+1) s_{34}-2-m=0 \\
2 s_{14}^{2}+\left((2 m+2) s_{34}-4-2 m\right) s_{14}+(-2-m) s_{34}+m+2=0 \\
\left(4+6 m+2 m^{2}\right) s_{34}^{2}+\left(-3-3 m^{2}-6 m\right) s_{34}+2 m+m^{2}=0 .
\end{array}\right.
$$

For all other $m$-values the simpler systems have no real solutions.

The case $m=1$ is straightforward, since $T_{1}$ and $T_{2}$ each have a unique solution corresponding to an equilateral triangle with either vortex 3 or 4 at the center. The case $m=0$ is uninteresting and we do not analyze it. It remains to study the solutions of system $T_{5}$.

- Solutions of $T_{5}$ with $s_{34}>0$ and $s_{14}>0$

The first two equations of $T_{5}$ are linear in $s_{12}$ and $s_{13}$, so that to each solution $\left(s_{14}, s_{34}\right)$ of the last two equations correspond one solution $\left(s_{12}, s_{13}, s_{14}, s_{34}\right)$ of $T_{5}$, provided that the determinant of the matrix of the coefficients of the linear system is not zero. Hence, we focus our attention on the last two equations of $T_{5}$. Let $A=2\left(2+3 m+m^{2}\right), B=$ $-3(m+1)^{2}$, and $C=m(2+m)$. Then the last equation of $T_{5}$ can be written as

$$
q_{1}\left(s_{34}\right)=A s_{34}^{2}+B s_{34}+C=0
$$

and can be viewed as a parametric equation of a parabola. Clearly $B<0$ for all $m \neq-1$, and $A>0$ for $m \in(-\infty,-2) \cup(-1, \infty)$. Thus $A>0, B<0$ on the domain where $T_{5}$ is valid. The vertex of the parabola has coordinates

$$
\left(-\frac{B}{2 A}, q_{1}\left(-\frac{B}{2 A}\right)\right)=\left(\frac{3(m+1)^{2}}{2\left(2+3 m+m^{2}\right)},-\frac{1}{8} \frac{(m-1)\left(m^{2}-4 m-9\right)}{m+2}\right) .
$$


It is easy to see that $-\frac{B}{2 A}>0$ and $q_{1}\left(-\frac{B}{2 A}\right)<0$ in the interval of interest, and hence the equation has two real solutions for each value of $m \in(-\infty,-2) \cup(-1 / 2,0) \cup(0,1)$. Since $C>0$ for $m \in(-\infty,-2) \cup(0, \infty)$ and $C<0$ for $m \in(-2,0)$, it follows that the equation has two positive solutions for $m \in(-\infty,-2) \cup(0,1)$ and only one positive solution for $m \in(-1 / 2,0)$.

Let us denote as $\beta_{1}$ and $\beta_{2}$ (with $\beta_{1}<\beta_{2}$ ) the (real) solutions of $q_{1}\left(s_{34}\right)=0$. Then through a standard analysis it is possible to find bounds for $\beta_{1}$ and $\beta_{2}$. Such bounds are summarized in the table below.

\begin{tabular}{|c|c|c|c|}
\hline solutions & $m \in(-\infty,-2)$ & $m \in\left(-\frac{1}{2}, 0\right)$ & $m \in(0,1)$ \\
\hline \hline$\beta_{1}$ & $0<\beta_{1}<\frac{1}{2}$ & $\beta_{1}<0$ & $0<\beta_{1}<\frac{1}{2}$ \\
\hline$\beta_{2}$ & $\beta_{2}>1$ & $0<\beta_{2}<1$ & $0<\beta_{2}<1$ \\
\hline
\end{tabular}

Now consider the third equation of system $T_{5}$. Let $A^{\prime}=2, B^{\prime}=\left(2(m+1) s_{34}-4-2 m\right)$, and $C^{\prime}=(m+2)\left(1-s_{34}\right)$. Then this equation takes the form

$$
q_{2}\left(s_{14}\right)=A^{\prime} s_{14}^{2}+B^{\prime} s_{14}+C^{\prime}=0 .
$$

Since this is a quadratic equation in $s_{14}$, there are going to be two solutions for each $s_{34}$ and $m$. We will denote the (real) solutions corresponding to $s_{34}=\beta_{1}$ as $\beta_{1}^{1}$ and $\beta_{1}^{2}$ (with $\beta_{1}^{1}<\beta_{1}^{2}$ ), and the ones corresponding to $s_{34}=\beta_{2}$ as $\beta_{2}^{1}$ and $\beta_{2}^{2}$ (with $\beta_{2}^{1}<\beta_{2}^{2}$ ). The vertex of this parabola has coordinates

$$
\begin{aligned}
& \left(-\frac{B^{\prime}}{2 A^{\prime}}, q_{2}\left(-\frac{B^{\prime}}{2 A^{\prime}}\right)\right)= \\
& \quad=\left(\frac{(m+1)\left(1-s_{34}\right)+1}{2},-\frac{1}{2}(m+1)^{2} s_{34}^{2}+m(m+2) s_{34}-\frac{m}{2}(m+2)\right)
\end{aligned}
$$

We can then view the ordinate of the vertex, denoted as $r\left(s_{34}\right)$, as a parabola with coefficients in $m$ :

$$
r\left(s_{34}\right)=-\frac{1}{2}(m+1)^{2} s_{34}^{2}+m(m+2) s_{34}-\frac{m}{2}(m+2)=A^{\prime \prime} s_{34}^{2}+B^{\prime \prime} s_{34}+C^{\prime \prime}=0 .
$$

The coordinates of the vertex of $r\left(s_{34}\right)$ are

$$
\left(-\frac{B^{\prime \prime}}{2 A^{\prime \prime}}, r\left(-\frac{B^{\prime \prime}}{2 A^{\prime \prime}}\right)\right)=\left(\frac{m(m+2)}{(m+1)^{2}},-\frac{1}{2} \frac{m(m+2)}{(m+1)^{2}}\right) .
$$

CASE I: $m \in(0,1)$

Since $-\frac{B^{\prime \prime}}{2 A^{\prime \prime}}>0, r\left(-\frac{B^{\prime \prime}}{2 A^{\prime \prime}}\right)<0$, and $A^{\prime \prime}<0$ for $m \in(0,1)$, it follows that $q_{2}\left(-\frac{B^{\prime}}{2 A^{\prime}}\right)<0$ for $m \in(0,1)$. Consequently, since $C^{\prime}>0$ in the same interval, $q_{2}=0$ has two positive solutions corresponding to each solution of $q_{1}=0$ for $m \in(0,1)$. In other words, there are four (positive) possible values for $s_{14}$, namely $\beta_{1}^{1}>0, \beta_{1}^{2}>0, \beta_{2}^{1}>0$, and $\beta_{2}^{2}>0$. 
CASE II: $m \in\left(-\frac{1}{2}, 0\right)$

For $m \in\left(-\frac{1}{2}, 0\right)$, we have $A^{\prime}>0$ and $C^{\prime}>0$. This implies that corresponding to $s_{34}=\beta_{2}$, there are either two real positive solutions or no real solutions. We want to show that there are two positive real solutions. Clearly $-\frac{B^{\prime \prime}}{2 A^{\prime \prime}}<0, r\left(-\frac{B^{\prime \prime}}{2 A^{\prime \prime}}\right)>0, A^{\prime \prime}<0$ and $C^{\prime \prime}>0$ for $m \in\left(-\frac{1}{2}, 0\right)$, so that $r=0$ has one positive and one negative root. If we show that the positive root is less than $s_{34}=\beta_{2}$, then it follows that $q_{2}\left(-\frac{B^{\prime}}{2 A^{\prime}}\right)<0$, and consequently $q_{2}=0$ has two positive solutions when $s_{34}=\beta_{2}$ (i.e., $\beta_{2}^{1}>0$ and $\beta_{2}^{2}>0$ ).

Subtracting $\beta_{2}$ from the positive root of $r=0$ yields

$$
\Delta=\frac{-\left(-m^{3}-7 m^{2}-4 x m+y m-7 m-8 x+y+3\right)}{4(m+2)(m+1)^{2}}
$$

where $x=\sqrt{-m(m+2)}$, and $y=\sqrt{(m-1)(m+1)\left(m^{2}-4 m-9\right)}$. Numerically it is easy to see that $\Delta<0$ for $m \in\left(-\frac{1}{2}, 0\right)$. However, we proceed more rigorously. Taking the numerator of the expression above and squaring the expressions for $x$ and $y$ yields three polynomials. Computing a lex Gröbner basis for these three polynomials, we obtain

$$
m\left(5 m^{3}+7 m^{2}+3 m+9\right)(m+2)^{2}(m+1)^{2}
$$

as one of the basis elements. It is trivial to see that this polynomial has no roots in $\left[-\frac{1}{2}, 0\right]$, that $\Delta(0)<0$, and that $\Delta\left(-\frac{1}{2}\right)<0$. Since $\Delta$ is continuous on $[-1 / 2,0]$, we deduce that $\Delta<0$ for $m \in\left(-\frac{1}{2}, 0\right)$, as desired.

CASE III: $m \in(-\infty,-2)$

If $s_{34}=\beta_{1}$ and $m \in(-\infty,-2)$, then $C^{\prime}<0$. Since $A^{\prime}>0$, it follows that $q_{2}=0$ has one positive and one negative real solutions corresponding to $\beta_{1}$. If $s_{34}=\beta_{2}$ and $m \in(-\infty,-2)$, then $C^{\prime}>0$. Since $r\left(-\frac{B^{\prime \prime}}{2 A^{\prime \prime}}\right)<0$ and $A^{\prime \prime}<0$ it follows that $q_{2}\left(-\frac{B^{\prime}}{2 A^{\prime}}\right)<0$. This together with the fact that $-\frac{B^{\prime}}{2 A^{\prime}}>0, A^{\prime}>0$ and $C^{\prime}>0$ implies that, corresponding to $\beta_{2}, q_{2}=0$ has two real positive solutions, (i.e., $\beta_{2}^{1}>0$, and $\beta_{2}^{2}>0$ ).

- Solutions of $T_{5}$ with $s_{34}>0, s_{14}>0$, and $s_{13}>0$

We now study which solutions among the ones found above have $s_{13}>0$. The resultant of the second and third equations of $T_{5}$ with respect to $s_{12}$ is

$$
R=2 s_{13}^{2}+\left((2 m+2) s_{34}-2 m-4\right) s_{13}+(-2-m) s_{34}+m+2 .
$$

If $s_{13}$ is replaced with $s_{14}$, this polynomial transforms into the one that appears in the third equation of $T_{5}$. Hence our discussion regarding $s_{14}$ applies to $s_{13}$. Note that since we saturated with respect to $s_{13}-s_{14}$, every solution must have $s_{13} \neq s_{14}$. Since $R=0$ has two solutions for each $m$ and $s_{34}$, when $s_{14}$ is one such solution, $s_{13}$ must be the other.

- Solutions of $T_{5}$ with $s_{34}>0, s_{14}>0, s_{13}>0$, and $s_{12}>0$

We now study which solutions among the ones found above have $s_{12}>0$. Consider the first equation of $T_{5}$ and rewrite it in the form

$$
s_{12}=\left(m^{2}+2 m\right) s_{34}+1-m^{2} .
$$




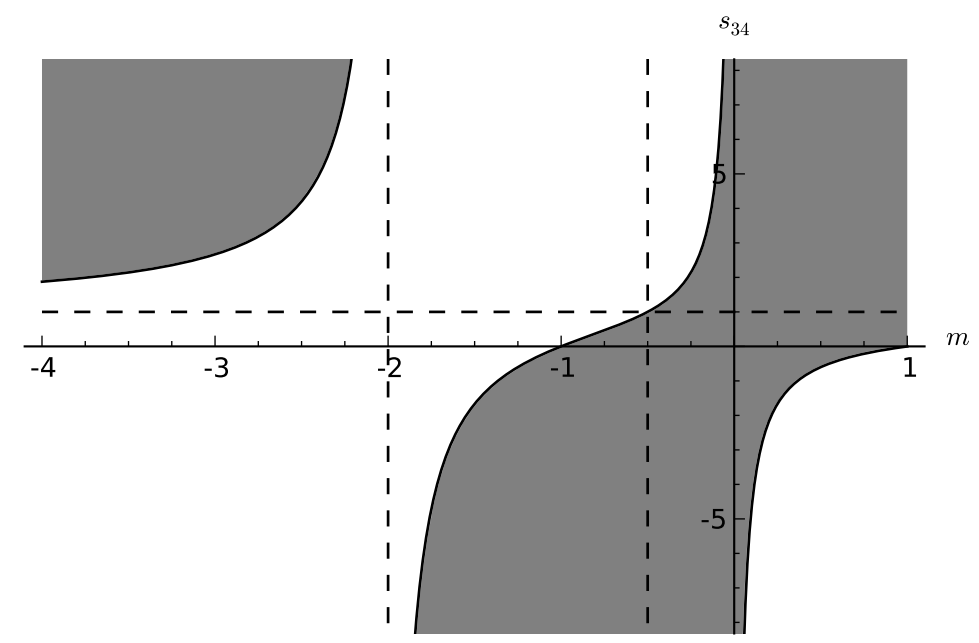

Figure 3: This figure summarizes the analysis of the sign of $s_{12}$. The grey areas represent regions where $s_{12}>0$. The white areas represent regions where $s_{12}<0$. Along the solid lines $s_{12}=0$.

This equation has a unique solution for each value of $s_{34}$ and $m$. Solutions corresponding to $s_{34}=\beta_{i}$ will be denoted $\alpha_{i}$. We can view this expression as defining a function of the two variables $m$ and $s_{34}$. The sign of this function, i.e., the sign of $s_{12}$, is summarized in Figure 3. As mentioned earlier, the last equation of $T_{5}$ gives that $s_{34}=\beta_{1}$ or $s_{34}=\beta_{2}$, and when $\beta_{i}>0$, we have $0<s_{34}<1$ for $m \in\left(-\frac{1}{2}, 0\right) \cup(0,1)$. Hence $s_{12}>0$ for any positive solution of $q_{1}\left(s_{34}\right)=0$.

The situation is a bit more delicate when $m \in(-\infty,-2)$. In this case, $\beta_{2}>1$, and thus we cannot arrive at a definitive conclusion just looking at Figure 3 . However, using standard methods, one can prove that the following function of $m$, defined by

$$
d(m)=\beta_{2}(m)-\frac{m^{2}-1}{m(m+2)},
$$

is always positive (where the graph of $\hat{f}(m)=\frac{m^{2}-1}{m(m+2)}$ is the boundary of the grey region in Figure 3 in the interval $(-\infty,-2))$. Thus, $\beta_{2}$ lies in the region where $s_{12}>0$ when $m \in(-\infty,-2)$. Consequently, there are always positive solutions of $T_{5}$ corresponding to $\beta_{2}$. On the other hand, $0<\beta_{1}<\frac{1}{2}$ for $m \in(-\infty,-2)$, and thus there are no positive solutions of $T_{5}$ corresponding to $\beta_{1}$ in this interval.

In summary, we have shown that the solutions $\left(s_{12}, s_{13}, s_{14}, s_{34}\right)^{T}$ with all components 
positive have the form

$$
\left[\begin{array}{l}
\alpha_{1} \\
\beta_{1}^{2} \\
\beta_{1}^{1} \\
\beta_{1}
\end{array}\right], \quad\left[\begin{array}{l}
\alpha_{1} \\
\beta_{1}^{1} \\
\beta_{1}^{2} \\
\beta_{1}
\end{array}\right], \quad\left[\begin{array}{l}
\alpha_{2} \\
\beta_{2}^{2} \\
\beta_{2}^{1} \\
\beta_{2}
\end{array}\right], \quad\left[\begin{array}{l}
\alpha_{2} \\
\beta_{2}^{1} \\
\beta_{2}^{2} \\
\beta_{2}
\end{array}\right],
$$

for $m \in(0,1)$, while they have the form

$$
\left[\begin{array}{l}
\alpha_{2} \\
\beta_{2}^{2} \\
\beta_{2}^{1} \\
\beta_{2}
\end{array}\right], \quad\left[\begin{array}{l}
\alpha_{2} \\
\beta_{2}^{1} \\
\beta_{2}^{2} \\
\beta_{2}
\end{array}\right]
$$

for $m \in(-\infty,-2) \cup\left(-\frac{1}{2}, 0\right)$.

We are left with deciding which solutions are convex and which are concave. For $m \in(0, \infty)$, it follows from the main result in [3] that, if the kite is convex, it must be a rhombus. Since the rhombus case was excluded, these solutions correspond to concave configurations. For $m \in\left(-\frac{1}{2}, 0\right)$, we have that $s_{34}=\beta_{2}<1$. However, according to Proposition 2.2, since $\lambda^{\prime}=-1$, the interior side connecting equal vorticities in a concave configuration is greater than 1 . Hence, the configurations are, in this case, convex. For $m \in(-\infty,-2)$ we have that $s_{34}=\beta_{2}>1$. Furthermore, substituting $s_{34}=\beta_{2}(m)$ into equation (30), we obtain an expression that is function of $m$ alone. Examining this function, it is easy to show that $s_{12}=\alpha_{2}(m)<1$ for all $m \in(-\infty, 0)$. Thus, from Propositions 2.2 and 2.4, it follows that the solutions correspond, in this case, to concave configurations.

This completes the proof of Lemma 7.3 .

We now consider the other case, where vortices 1 and 2 lie on the axis of symmetry. We compute a Gröbner basis for the ideal $\left\langle\overline{\mathcal{F}}^{\prime}, \overline{\mathcal{G}}^{\prime}, \bar{e}_{C M}^{\prime}, \bar{H}^{\prime}\right\rangle$, where $\overline{\mathcal{F}}^{\prime}, \overline{\mathcal{G}}^{\prime}, \bar{e}_{C M}^{\prime}, \overline{\mathcal{H}}^{\prime}$ are obtained from $\widetilde{\mathcal{F}}, \widetilde{\mathcal{G}}, \widetilde{e}_{C M}$ and $\widetilde{\mathcal{H}}$, respectively, by imposing the conditions $s_{14}=s_{13}$ and $s_{24}=s_{23}$. We saturate with respect to $s_{13}-s_{23}$ in order to exclude the rhombus configurations. We also saturate with respect to $s_{12}, s_{13}, s_{23}, s_{34}$ and $m$. This computation yields a polynomial system that we will call $G_{1}$. Then we can prove the following lemma.

Lemma 7.4. For each value of $m \in(1, \infty)$, system $G_{1}$ has exactly four real positive solutions corresponding to concave configurations. For each value of $m \in(-\infty,-2) \cup$ $\left(-\frac{1}{2}, 0\right)$, system $G_{1}$ has exactly two real positive solutions. Such solutions correspond to concave configurations if $m \in\left(-\frac{1}{2}, 0\right)$, and to convex configurations if $m \in(-\infty,-2)$. For $m=1$, system $G_{1}$ has exactly two real positive solutions corresponding to an equilateral triangle with either vortex 1 or 2 at the center. There are no positive real solutions for other values of $m$. 
Proof. Computing a full triangular decomposition of $G_{1}$, with the positivity conditions $s_{12}>0, s_{13}>0, s_{14}>0$ and $s_{34}>0$, we decompose $G_{1}$ into three simpler systems $S_{1}, S_{2}$ and $S_{3}$. For $m=1$, we have two systems, $S_{1}$ and $S_{2}$. They are linear, and each of them has a unique solution corresponding to an equilateral triangle configuration with vortex 1 or 2 at the center. For $m \in(-\infty,-2) \cup\left(-\frac{1}{2}, 0\right) \cup(1, \infty)$, we obtain a third system $S_{3}$. This last system, possibly after computing a lexicographic Gröbner basis, can be reduced to $T_{5}$ by performing the transformation $\left(m, s_{12}, s_{13}, s_{23}, s_{34}\right) \mapsto\left(\frac{1}{m}, s_{34}, s_{14}, s_{13}, s_{12}\right)$ and clearing the denominators. This transforms each of the four polynomial equations in $S_{3}$ to the equations of $T_{5}$. Consequently, these cases follow from Lemma 7.3. For all other $m$-values the simpler systems have no real solutions.

Remark. 1. The proofs of Lemmas 7.3 and 7.4 yield some interesting information on the type of kite central configurations. For example, when $m \in(0,1)$, there are solutions only when the smaller strength vortices (3 and 4 ) are on the line of symmetry. In this case, there are two geometrically distinct configurations for each choice of the central vortex.

2. As described in the introduction, the equilateral triangle with a central vortex bifurcates into both concave kite configurations and asymmetric concave configurations as $m$ decreases through $m=1$. The fact that this particular solution shows up in both Sections 6.3 and 7.2 is hardly surprising. In fact, when solving the Albouy-Chenciner equations for $m=1$ without any restrictions on the variables, this solution occurs four times with a multiplicity of four. The Hessian matrix $D^{2}(H+\lambda I)$ evaluated at this solution has a null space of dimension 2 (excluding the "trivial" eigenvector in the direction of rotation). One can check that there are eigenvectors in the null space corresponding to both a symmetric and asymmetric perturbation of the solution. In fact, the symmetric perturbation gives rise to the concave kite configurations described above. The central configuration consisting of four equal vortices on an equilateral triangle with a vortex at the center is a highly degenerate solution.

\subsection{Kite configurations: $\lambda^{\prime}>0$}

In Section 2.1 we observed that, if the vorticities have different signs, we could have central configurations with $\lambda^{\prime}>0$. In this case, taking $\Gamma_{1}=\Gamma_{2}=1$ and $\Gamma_{3}=\Gamma_{4}=m<0$, the configuration is necessarily convex with vortices 1 and 2 on one diagonal and vortices 3 and 4 on the other. In this section, we first show that these convex solutions must contain a line of symmetry. Based on the main result in [3] (applicable to our problem only when $m>0$ ), we might expect each solution to contain two lines of symmetry, forming a rhombus. We show here that this is not necessarily true. When $\lambda^{\prime}>0$ and $m \in\left(m^{*},-1 / 2\right)$, where $m^{*} \approx-0.5951$, there exists a family of convex kite central configurations that are not rhombi. There does exist a family of rhombi when $\lambda^{\prime}>0$, as discussed in Section 7.4. In fact, this family of rhombi undergoes a pitchfork bifurcation at $m=m^{*}$ that results in the convex kites. 
Proposition 7.5. Let $\Gamma_{1}=\Gamma_{2}=1$ and $\Gamma_{3}=\Gamma_{4}=m<0$. Any strictly planar solution to the Albouy-Chenciner equations with $\lambda^{\prime}=1$ must possess a line of symmetry.

Proof. The type of computations required are very similar to those used in Sections 6.3 and 7.2. Consider the Albouy-Chenciner and the unsymmetrized Albouy-Chenciner equations together with the Cayley-Menger determinant and Dziobek equations with the normalization $\lambda^{\prime}=1$. Take a Gröbner basis of the ideal generated by such equations and saturate with respect to some of the variables to eliminate possible solutions where one of the mutual distances has zero length. Then, saturating with respect to the differences $\left(s_{13}-s_{24}\right),\left(s_{23}-s_{14}\right),\left(s_{13}-s_{14}\right),\left(s_{23}-s_{24}\right),\left(s_{13}-s_{23}\right)$ and $\left(s_{14}-s_{24}\right)$, yields a system of 12 polynomial equations. Analyzing this system by the method of triangular decomposition of semialgebraic systems (with the inequalities $s_{i j}>0$ ) yields an empty triangular system. Therefore, due to the saturation and the Symmetry Lemma, there are no asymmetric solutions in this case.

To find the solutions with $\lambda^{\prime}>0$, we impose the symmetry on the configuration and use equations (7) since the areas $A_{i}$ are easily computable. Suppose that vortices 1 and 2 are on the axis of symmetry and set $r_{14}=r_{13}$ and $r_{24}=r_{23}$. We impose the scaling $r_{34}=1$ rather than choose $\lambda^{\prime}=1$. Introduce two new variables $x$ and $y$ measuring the distance between the intersection of the two diagonals and vortices 1 and 2 , respectively. Thus, we have the simple geometric equations

$$
x^{2}+\frac{1}{4}=r_{13}^{2}, \quad y^{2}+\frac{1}{4}=r_{23}^{2} \quad \text { and } \quad x+y=r_{12} .
$$

Equations (31) taken together with $r_{34}=1$ imply that the Cayley-Menger determinant $e_{C M}$ vanishes.

Under this setup, convex configurations correspond to $x>0$ and $y>0$, while concave configurations have $x y<0$. The oriented areas of the four triangles are given by $A_{1}=y / 2$, $A_{2}=x / 2$ and $A_{3}=A_{4}=-r_{12} / 4$, where the signs are taken without loss of generality. Note that one of the Dziobek equations in (8) is automatically satisfied. The other equation yields an expression for $\lambda^{\prime}$ given by

$$
\lambda^{\prime}=\frac{r_{13}^{2} r_{23}^{2}-r_{12}^{2}}{r_{12}^{2}\left(r_{13}^{2}+r_{23}^{2}\right)-r_{13}^{2} r_{23}^{2}\left(r_{12}^{2}+1\right)} .
$$

Equations (7), the formula for $\lambda^{\prime}$ and equations (31) yield a polynomial system in the variables $r_{12}, r_{13}, r_{23}, x, y, \lambda^{\prime}$ and $m$. We then saturate this system with respect to $r_{13}, r_{23}, r_{13}-$ $r_{23}$ and $r_{13}+r_{23}$ to eliminate the rhombus solutions and insure the mutual distance variables are nonzero. Denote the resulting polynomial system as $P_{\text {kite }}$. Analyzing Gröbner bases of the ideal generated by $P_{\text {kite }}$ with different elimination orderings yields the following theorem. Recall that $m^{*} \approx-0.5951$ is the only real root of the cubic $9 m^{3}+3 m^{2}+7 m+5$.

Theorem 7.6. For $m^{*}<m<-1 / 2$, there exists four convex kite configurations with vortices 1 and 2 on the axis of symmetry. These solutions have $\lambda^{\prime}>0$ and are not rhombi. There are no other strictly kite solutions (with vortices 1 and 2 on the axis of symmetry) with $\lambda^{\prime}>0$ and $m<0$. 
Proof. Let $G_{\text {kite }}$ be the Gröbner basis of the ideal generated by $P_{\text {kite }}$ with respect to the lex order where $r_{23}>r_{13}>r_{12}>\lambda^{\prime}>y>x>m$. The first element in $G_{\text {kite }}$ is an even 8th-degree polynomial in $x$ with coefficients in $m$. Letting $z=x^{2}$, this polynomial is given by

$$
\begin{aligned}
\zeta_{m}(z)= & 256 m^{2}(m+2)(2 m+1)^{2} z^{4}-256 m\left(9 m^{4}+23 m^{3}+17 m^{2}-m-3\right) z^{3} \\
& +\left(1728 m^{5}+3136 m^{4}+992 m^{3}-384 m^{2}+64 m+128\right) z^{2} \\
& +\left(-432 m^{5}-336 m^{4}+48 m^{3}-80 m^{2}+16 m+64\right) z+(m+2)^{3} .
\end{aligned}
$$

Computing a Gröbner basis with the same ordering of variables except that $x>y>m$ yields the same polynomial with $z=y^{2}$. The discriminant of $\zeta_{m}$ is a positive multiple of

$$
m^{4}(m+1)^{3}(2 m+1)\left(9 m^{3}+3 m^{2}+7 m+5\right)\left(9 m^{2}+4 m-1\right)^{2}(m-1)^{2}\left(q_{k}(m)\right)^{2}
$$

where $q_{k}(m)=2 m^{5}+8 m^{3}+14 m^{2}+4 m-1$. Note that the discriminant vanishes at $m=m^{*}$. It also vanishes at $m=-1 / 2$, where $\zeta_{m}(z)$ becomes a cubic polynomial with roots $3 / 4$ and $-1 / 4$ (multiplicity 2 ). It is straight-forward to check that the discriminant is strictly negative for $m^{*}<m<-1 / 2$. Consequently, $\zeta_{m}(z)$ has precisely two real roots for each $m$-value in this interval.

To see that these roots are always positive, we first use resultants to compute the repeated root of $\zeta_{m}(z)$ when $m=m^{*}$. This yields the value $z^{*} \approx 1.9566$, the only root of the cubic $c_{u}(z)=320 z^{3}-656 z^{2}+60 z-3$. Next consider the ideal in $\mathbb{C}[z, m]$ generated by the system $\left\{\zeta_{m}, c_{u}\right\}$, which has $\left(z^{*}, m^{*}\right)$ in its variety. Computing a Gröbner basis for this ideal which eliminates $z$, we obtain a polynomial in $m$ that has no roots for $m^{*}<m \leq-1 / 2$. Since $\zeta_{m}\left(z^{*}\right)<0$ when $m=-1 / 2$, it follows that $\zeta_{m}\left(z^{*}\right)<0$ for any $m \in\left(m^{*},-1 / 2\right)$. Then, since the constant term and leading coefficient of $\zeta_{m}$ are both positive for $m^{*}<m<-1 / 2$, the two real roots of $\zeta_{m}$ must be positive.

Choose $x$ to be the positive square root of one of the roots of $\zeta_{m}$. The second element in the Gröbner basis $G_{\text {kite }}$ is linear in $y$, and the coefficient of $y$ is non-zero for $m^{*}<m<-1 / 2$. Thus, by the Extension Theorem, we can extend a solution $(x, m)$ of $\zeta_{m}(x)=0$ to a unique partial solution $(y, x, m)$, where $y$ must be real. By considering the other polynomials in $G_{\text {kite }}$, three of which are given by equations (31), the Extension Theorem repeatedly applies to extend $(y, x, m)$ to a unique full solution of system $P_{\text {kite }}$ where $x, r_{12}, r_{13}, r_{23}$ are all positive and $r_{13} \neq r_{23}$. As expected, many of the elements in $G_{\text {kite }}$ featuring the saturation variable for $r_{13}-r_{23}$ have a leading coefficient that is a multiple of the key cubic $9 m^{3}+3 m^{2}+7 m+5$.

To see that $\lambda^{\prime}$ is positive, we compute a Gröbner basis for $P_{\text {kite }}$ which eliminates all variables except $\lambda^{\prime}$ and $m$. The first element in this Gröbner basis, a quadratic polynomial in $\lambda^{\prime}$ with coefficients in $m$, is

$$
2 m^{2}(m+1) \lambda^{\prime 2}+(4 m-1)(m+1)^{2} \lambda^{\prime}+m(m+2)(2 m+1) .
$$

It is straight-forward to show that the roots of this quadratic are real and positive for $m^{*}<m<-1 / 2$. Consequently, $\lambda^{\prime}>0$ and the configuration corresponding to the 
solution guaranteed by the Extension Theorem is convex. Therefore, $y>0$ is also assured, and by symmetry, $y$ must be the positive square root of the remaining real root of $\zeta_{m}$. Since we have saturated to eliminate the rhombus solutions, we must have $x \neq y$. Thus, there are two solutions: one where $x>\sqrt{z^{*}}>y>0$, and one where $y>\sqrt{z^{*}}>x>0$. The full count of four solutions comes from reflecting each of these solutions about the axis of symmetry (or simply interchanging vortices 3 and 4). This proves the first part of the theorem.

To see that there are no other solutions (other than a rhombus) with $\lambda^{\prime}>0$, first consider the case $m \in(-1 / 2,0)$. According to equation (32), the two possible choices for $\lambda^{\prime}$ are real, with opposite sign. Denote the larger, positive root as $\lambda_{+}^{\prime}$ and suppose there was a real solution to system $P_{\text {kite }}$ with $\lambda^{\prime}=\lambda_{+}^{\prime}>0$. Since the quadratic (32) is strictly negative when $\lambda^{\prime}=3$ and $m \in(-1 / 2,0)$, we can assume that $\lambda_{+}^{\prime}>3$. Next, the top two equations in (7) imply that

$$
4 m^{2} x y\left(1+\lambda^{\prime}\right)=1+\lambda^{\prime} r_{12}^{2}
$$

If our real solution has $\lambda^{\prime}>0$, then it is convex and $x y>0$. Since $\lambda_{+}^{\prime}>3$, we have that

$$
1+\lambda_{+}^{\prime}\left(x^{2}+y^{2}\right)+x y\left(\lambda_{+}^{\prime}-1\right)>0 .
$$

Taking $m \in(-1 / 2,0)$, this in turn implies that

$$
4 m^{2} x y\left(1+\lambda_{+}^{\prime}\right)<x y\left(1+\lambda_{+}^{\prime}\right)<1+\lambda_{+}^{\prime}(x+y)^{2},
$$

which violates equation (33). Therefore, any real solution for $m \in(-1 / 2,0)$ must have $\lambda^{\prime}<0$.

Recall that for $m=-1 / 2, \zeta_{m}$ reduces to a cubic polynomial with only one positive root at $\zeta=3 / 4$. Therefore, the only possible solution to system $P_{\text {kite }}$ at $m=-1 / 2$ has $x=y=\sqrt{3} / 2$, a rhombus solution. At $m=m^{*}, \zeta_{m}$ has only one positive root at $z^{*}$ (multiplicity two), which also implies that $x=y$. For the case $-1<m<m^{*}$, the discriminant of $\zeta_{m}$ is positive; however, using Lemma 3.5 it is straight-forward to check that all the roots of $\zeta_{m}$ are complex. Hence there are no real solutions to system $P_{\text {kite }}$ when $-1<m<m^{*}$.

For $m<-1$, the discriminant of $\zeta_{m}$ is negative so there are two real roots. To determine their sign, we note that $\zeta_{m}(-1 / 4)=128 m^{3}(m+1)(2 m+1)$ is strictly negative for $-2<m<-1$. Since both the leading coefficient and the constant term of $\zeta_{m}$ are positive when $-2<m<-1$, it follows that the real roots of $\zeta_{m}$ are negative in this case, and there are no real solutions to system $P_{\text {kite }}$. For $m<-2$, the signs of the leading coefficient and constant term both flip to become negative. Since $\zeta_{m}(3 / 4)=-128(m-1)(2 m+1)^{2}$ is strictly positive when $m<-2$, it follows that $\zeta_{m}$ has two positive real roots. However, examining the roots of the quadratic in equation (32), we see that $\lambda^{\prime}<0$ whenever $m<-2$. Finally, when $m=-2, \zeta_{m}$ reduces to a cubic with only one real root at zero. Thus, we have shown that for $m<0$ and $m \notin\left(m^{*},-1 / 2\right)$, there are either no real solutions or only solutions with $\lambda^{\prime}<0$. This completes the proof of Theorem 7.6. 
Remark. 1. Since Theorem 7.6 applies for all $m<0$, we do not need to consider the case with vortices 3 and 4 on the axis of symmetry. If there existed a solution for some $m \in(-1,0)$ having $\lambda^{\prime}>0$ and vortices 3 and 4 on the axis of symmetry, we could rescale the vortex strengths by $1 / m$ and relabel the vortices to obtain a solution with $m<-1, \lambda^{\prime}>0$, and vortices 1 and 2 on the axis of symmetry. But this contradicts the last statement of the theorem.

2. For completeness, we note that the cases having real, positive solutions with $\lambda^{\prime}<0$ agree with the results in Section 7.2. For example, when $-1 / 2<m<0$, we find concave kite configurations with vortices 1 and 2 on the axis of symmetry, as predicted by Theorem 7.2 . For $m<-2$, we find convex kite solutions with vortices 1 and 2 on the axis of symmetry. Rescaling the vortex strengths by $1 / m$ and relabeling the vortices gives a family of convex kites with vortices 3 and 4 on the axis of symmetry and $-1 / 2<m<0$. This also concurs with Theorem 7.2 .

\subsection{The rhombus solutions}

In this final section we study convex kite configurations that have two lines of symmetry, namely rhombus configuations. Here we have four congruent exterior sides, $r_{13}=r_{14}=$ $r_{23}=r_{24}$ with the diagonals satisfying the relation $r_{12}^{2}+r_{34}^{2}=4 r_{13}^{2}$. The areas satisfy $A_{1}=A_{2}=-A_{3}=-A_{4}$. The first and last equations in (9) are clearly satisfied. The middle four equations in (9) are equivalent. They, along with the value of $\lambda^{\prime}$ determined by (8), yield a formula for $m$ given by

$$
m=\frac{x^{2}\left(3-x^{2}\right)}{3 x^{2}-1}
$$

where $x=r_{34} / r_{12}$ is the ratio between the diagonals of the rhombus. Note that $m>0$ if and only if $1 / \sqrt{3}<x<\sqrt{3}$. As usual, we restrict to the case $-1<m \leq 1$.

Unlike the isosceles trapezoid case, here we have solutions for $m<0$. In fact, there are two geometrically distinct families of rhombi when $m<0$. This can be seen by inverting equation (34), which yields

$$
x^{2}=\frac{1}{2}\left(3-3 m \pm \sqrt{(3-3 m)^{2}+4 m}\right),
$$

where + is taken if $m>0$ and + or - may be chosen if $m<0$. Using equation (4), we compute that

$$
\lambda=\frac{4\left(m^{2}+4 m+1\right)}{r_{12}^{2}\left(2+3 m-3 m^{2} \pm m \sqrt{9 m^{2}-14 m+9}\right)},
$$

with the same sign choices as for $x^{2}$. Note that the numerator of $(36)$ vanishes at $m=$ $-2+\sqrt{3} \approx-0.2679$. The denominator also vanishes at this special value, but only when + is chosen. For the rhombus solution when + is taken in both (35) and (36), the value of the angular velocity $\lambda$ is always positive (thus $\lambda^{\prime}<0$ ) and monotonically increasing in $m$ for $m \in(-1,1]$. In contrast, for the rhombus solution when - is taken in both (35) 


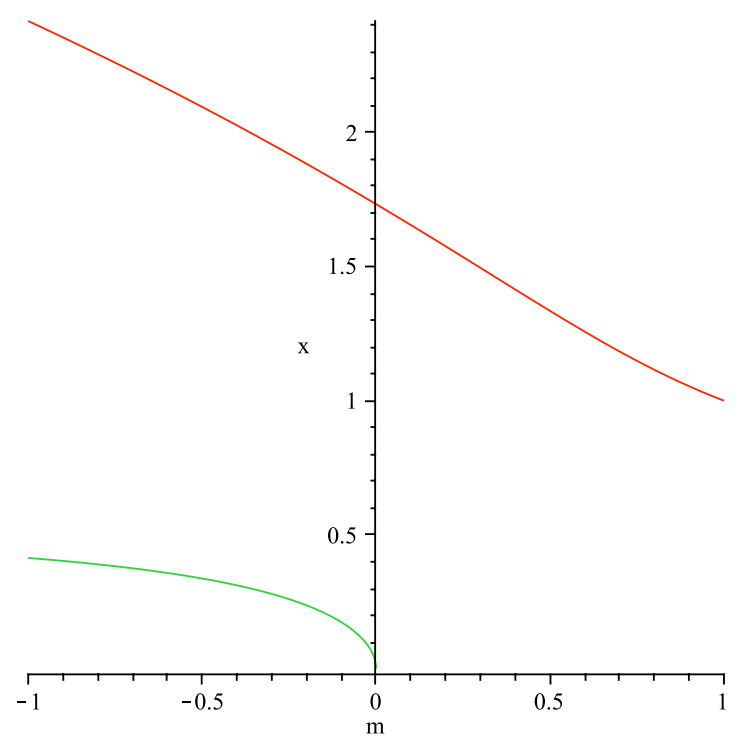

Figure 4: A plot of $x$ versus $m$ for the two rhombus families, where $x=r_{34} / r_{12}$ is the ratio between the diagonals of the rhombus.

and 36 ( $m<0$ only), we have $\lambda>0$ (thus $\lambda^{\prime}<0$ ) only for $-2+\sqrt{3}<m<0$. At the special value $m=-2+\sqrt{3}$, the rhombus relative equilibrium actually becomes an equilibrium (see Section 4.1). As $m$ decreases through $-2+\sqrt{3}$, the direction of rotation flips, $\lambda$ becomes negative and $\lambda^{\prime}$ becomes positive. We summarize our conclusions in the following theorem.

Theorem 7.7. There exists two one-parameter families of rhombi relative equilibria with vortex strengths $\Gamma_{1}=\Gamma_{2}=1$ and $\Gamma_{3}=\Gamma_{4}=m$. The vortices 1 and 2 lie on opposite sides of each other, as do vortices 3 and 4 . Let $\beta=3-3 \mathrm{~m}$. The mutual distances are given by

$$
\left(\frac{r_{34}}{r_{12}}\right)^{2}=\frac{1}{2}\left(\beta \pm \sqrt{\beta^{2}+4 m}\right) \text { and }\left(\frac{r_{13}}{r_{12}}\right)^{2}=\frac{1}{8}\left(\beta+2 \pm \sqrt{\beta^{2}+4 m}\right)
$$

describing two distinct solutions. Taking + in $($ (37) yields a solution for $m \in(-1,1]$ that always has $\lambda>0$. Taking - in (37) yields a solution for $m \in(-1,0)$ that has $\lambda>0$ for $m \in(-2+\sqrt{3}, 0)$, but $\lambda<0$ for $m \in(-1,-2+\sqrt{3})$. At $m=-2+\sqrt{3}$, the - solution becomes an equilibrium. The case $m=1$ reduces to the square. For $m>0$, the larger pair of vortices lie on the shorter diagonal.

Proof. The formula for $r_{13} / r_{12}$ comes from $1+x^{2}=4\left(r_{13} / r_{12}\right)^{2}$. For the case $m>0$, it is easy to show from equation (34) that $m<1$ if and only if $1<x<\sqrt{3}$. This verifies the last statement in the theorem.

Remark. 1. One way to visualize the two rhombus solutions is captured in Figure 4 , where a plot of $x=r_{34} / r_{12}$ versus $m$ is shown. Beginning with the square at $m=1$, 

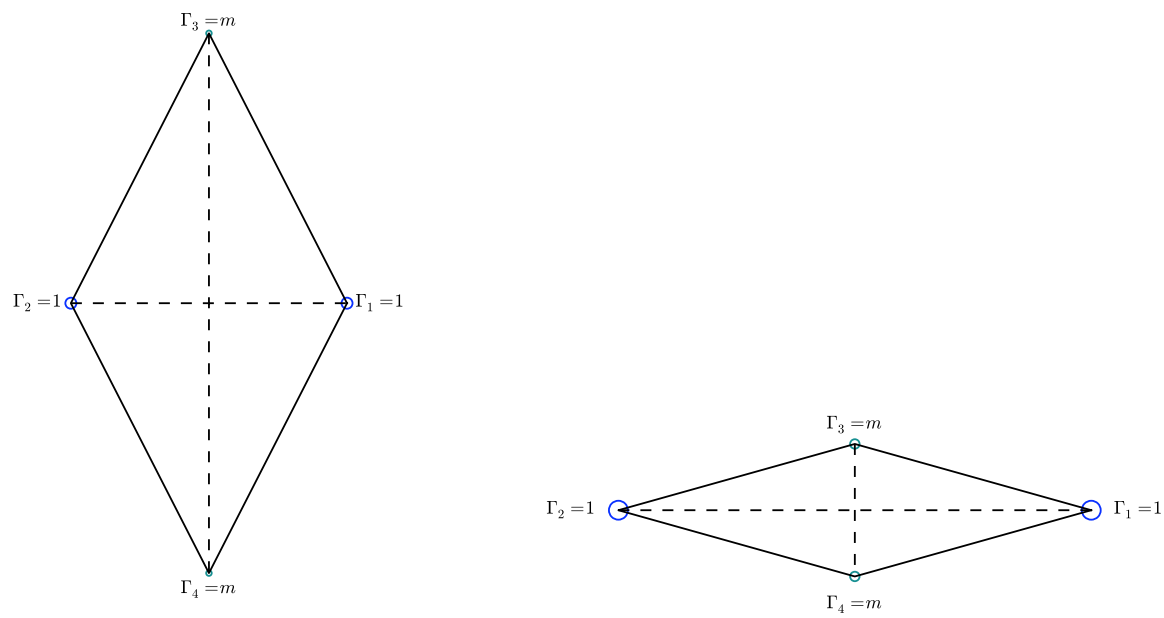

Figure 5: The two distinct rhombi relative equilibria when $m=-0.3$. The solutions rotate in opposite directions.

as $m$ decreases, the ratio of the diagonals of the rhombus increases from 1 to $\sqrt{3}$, with the larger pair of vortices lying on the shorter diagonal. At $m=0$ a bifurcation occurs, and a new family is born emerging out of a binary collision between vortices 3 and 4 . Unlike the previous family, which continues past the bifurcation, this family has the larger pair of vortices (in absolute value) on the longest diagonal (see Figure 5). As $m \rightarrow-1^{+}, x \rightarrow \sqrt{2}-1$ for the new family while $x \rightarrow \sqrt{2}+1$ for the previous family.

2. The rhombus solution when - is chosen in equation (37) undergoes a pitchfork bifurcation at the special parameter value $m^{*} \approx-0.5951$. As $m$ increases through $m^{*}$, the rhombus solution bifurcates into two convex kite solutions with the positive strength vortices on the axis of symmetry. The two kites are distinguished by whether $r_{13}>r_{23}$ or $r_{13}<r_{23}$. Since the rhombus solution continues to exist past the bifurcation, we have a pitchfork bifurcation. The Hessian matrix $D^{2}(H+\lambda I)$ evaluated at the rhombus solution at $m=m^{*}$ has a null space of dimension 1 (excluding the "trivial" eigenvector in the direction of rotation) and contains an eigenvector corresponding to a perturbation in the direction of the convex kite solution found in Section 7.3 .

3. The fact that there are two geometrically distinct rhombus solutions for $m<0$ (plus two convex kites when $m^{*}<m<-1 / 2$ ) indicates that there is not a unique (up to symmetry) convex central configuration for these four choices of vorticities. This contrasts with the Newtonian four-body problem where it is thought (although unproven) that there is a unique convex central configuration for any ordering of four positive masses.

Acknowledgements: Part of this work was carried out when the authors were visiting the American Institute of Mathematics in May of 2011. We gratefully acknowledge their 
hospitality and support. GR was also supported by a grant from the National Science Foundation and MS was supported by a NSERC Discovery Grant. 


\section{References}

[1] Alain Albouy, The symmetric central configurations of four equal masses, Hamiltonian dynamics and celestial mechanics (Seattle, WA, 1995), Contemp. Math., vol. 198, Amer. Math. Soc., Providence, RI, 1996, pp. 131-135.

[2] Alain Albouy and Alain Chenciner, Le problème des n corps et les distances mutuelles, Inventiones Mathematicae 131 (1997), no. 1, 151-184.

[3] Alain Albouy, Yanning Fu, and Shanzhong Sun, Symmetry of planar four-body convex central configurations, Proceedings of the Royal Society A: Mathematical, Physical and Engineering Sciences 464 (2008), no. 2093, 1355-1365.

[4] Hassan Aref, Point vortex dynamics: a classical mathematics playground, Journal of Mathematical Physics 48 (2007), no. 6, 065401, 23 pp.

[5] Jean F. Barros and Eduardo S. G. Leandro, The set of degenerate central configurations in the planar restricted four-body problem, SIAM Journal on Mathematical Analysis 43 (2011), no. 2, 634.

[6] Jacek Bochnak, Michel Coste, and Marie-Françoise Roy, Real algebraic geometry, Springer, September 1998.

[7] Gerolamo Cardano, Artis magnae, Johann Petreius, Nuremberg, 1545.

[8] Martin Celli, Sur les mouvements homographiques de $n$ corps associés à des masses de signe quelconque, le cas particulier où la somme des masses est nulle, et une application à la recherche de choréographies perverse, Ph.D. thesis, Université Paris 7, France, 2005.

[9] Changbo Chen, James H. Davenport, John P. May, Marc Moreno Maza, Bican Xia, and Rong Xiao, Triangular decomposition of semi-algebraic systems, Proceedings of the 2010 International Symposium on Symbolic and Algebraic Computation, ISSAC '10, ACM Press, 2010, pp. 187-194.

[10] Josep M. Cors and Gareth E. Roberts, Four-body co-circular central configurations, Nonlinearity 25 (2012), no. 2, 343-370.

[11] David A. Cox, John B. Little, and Donal O'Shea, Ideals, varieties, and algorithms: An introduction to computational algebraic geometry and commutative algebra, Springer, 2007.

[12] Wolfram Decker, Gert-Martin Greuel, Gerhard Pfister, and Hans Schönemann, Singular 3-1-3 - a computer algebra system for polynomial computations, (2011), http://www.singular.uni-kl.de.

[13] Otto Dziobek, Über einen merkwürdigen fall des vielkörperproblems, Astronomische Nachrichten 152 (1900), 33. 
[14] Marshall Hampton, Concave central configurations in the four-body problem, Ph.D. thesis, University of Washington, Seattle, 2002.

[15] Marshall Hampton and Richard Moeckel, Finiteness of relative equilibria of the fourbody problem, Inventiones Mathematicae 163 (2005), no. 2, 289-312.

[16] _ Finiteness of stationary configurations of the four-vortex problem, Transactions of the American Mathematical Society 361 (2009), no. 3, 1317-1332.

[17] Gustav Kirchhoff, Vorlesungen über mathematische physik, B.G. Teubner, 1883.

[18] Kenneth Meyer and Dieter Schmidt, Bifurcations of relative equilibria in the $n$-body and Kirchhoff problems, SIAM Journal on Mathematical Analysis 19 (1988), no. 6, 1295-1313.

[19] Kenneth R. Meyer, Glen R. Hall, and Dan Offin, Introduction to Hamiltonian dynamical systems and the n-body problem, second ed., Springer, 2009.

[20] Forest Ray Moulton, The straight line solutions of the problem of $n$ bodies, The Annals of Mathematics 12 (1910), no. 1, 1-17.

[21] Kevin Anthony O'Neil, Stationary configurations of point vortices, Transactions of the American Mathematical Society 302 (1987), no. 2, 383-425.

[22] Julian Palmore, Relative equilibria of vortices in two dimensions, Proceedings of the National Academy of Sciences of the U.S.A. 79 (1982), no. 2, 716-718.

[23] Ernesto Perez-Chavela and Manuele Santoprete, Convex four-body central configurations with some equal masses, Archive for Rational Mechanics and Analysis 185 (2007), no. 3, 481-494.

[24] Dieter Schmidt, Central configurations and relative equilibria for the n-body problem, Classical and celestial mechanics (Recife, 1993/1999), Princeton Univ. Press, Princeton, NJ, 2002, p. 1-33.

[25] Silke Spang, A zero-dimensional approach to compute real radicals, The Computer Science Journal of Moldava 16 (2008), no. 1, 64-92.

[26] _ realrad.1ib. A singular 3-1-3 library for computing real radicals, 2011.

[27] William A. Stein et al., Sage mathematics software (Version 4.6.2), 2011, http://www.sagemath.org. 